\title{
The nuclear interaction at Oklo 2 billion years ago
}

\author{
Yasunori Fujii \\ Nihon Fukushi University, Handa, Aichi, 475-0012 Japan \\ and \\ Institute of Cosmic Ray Research (ICRR), University of Tokyo, \\ Tanashi, Tokyo, 188-8502 Japan \\ Akira Iwamoto, Tokio Fukahori, Toshihiko Ohnuki, Masayuki Nakagawa \\ Japan Atomic Energy Research Institute (JAERI) \\ Tokai-mura, Naka-gun, Ibaraki, 319-1195 Japan \\ Hiroshi Hidaka \\ Department of Earth and Planetary Systems Science, Hiroshima University \\ Higashi-Hiroshima, Hiroshima, 739-8526 Japan \\ Yasuji Oura \\ Department of Chemistry, Tokyo Metropolitan University, \\ Hachioji, Tokyo, 192-0397 Japan \\ Peter Möller \\ Theoretical Division, Los Alamos National Laboratory, New Mexico 87545, USA
}

\begin{abstract}
We re-examine the effort to constrain the time-variability of the coupling constants of the fundamental interactions by studying the anomalous isotopic abundance of Sm observed at the remnants of the natural reactors which were in operation at Oklo about 2 billion years ago, in terms of a possible deviation of the resonance energy from the value observed today. We rely on new samples that were carefully collected to minimize natural contamination and also on a careful temperature estimate of the reactors. We obtain the upper bound $(-0.2 \pm 0.8) \times 10^{-17} \mathrm{y}^{-1}$ on the fractional rate of change of the electromagnetic as well as the strong interaction coupling constants. Our result basically agrees with and even suggests some improvement of the result due recently to Damour and Dyson. Strictly speaking, however, we find another range of the resonance energy shift indicating a nonzero time variation of the constants. We find a rather strong but still tentative indication that this non-null range can be ruled out by including the Gd data, for which it is essential to take the effect of contamination into account.
\end{abstract}

${ }^{*}$ A preliminary report is found in Ref. [1]. 


\section{Introduction}

Few aspects of physics can be understood without recourse to fundamental "constants," such as the speed of light $c$, Planck's constant $h$, Newton's constant $G$, the fine-structure constant $\alpha$, and its QCD counterpart $\alpha_{\mathrm{QCD}}$, or the pion-nucleon coupling constant squared $\alpha_{\mathrm{s}}=g_{\pi N}^{2} / 4 \pi$ in most of realistic analyses. To this list one may also add the masses of certain fundamental particles, like the quarks and the leptons, or some of the vacuum expectation values, and perhaps the cosmological constant $\Lambda$. The traditional assumption that these parameters of physics theories are constant was challenged for the first time by Dirac, who suggested that $G$ might vary as $t^{-1}$ with $t$ the cosmic time [2]. This paper inspired to many probes of the possible time variability of various fundamental constants.

However, no positive evidence for any time-dependence has been discovered so far, nor does Dirac's original argument seem fully convincing. Nevertheless, the very idea that at least some of the fundamental constants may not be truly constant still attracts serious attention partly because this appears to be a rather general outcome of the recent efforts toward unifying particle physics and gravity.

Quite remarkable is the finding that the upper bounds obtained so far for $\alpha$ and $\alpha_{\mathrm{s}}$ are many orders of magnitude below the value $\sim 10^{-10} \mathrm{y}^{-1}$ expected naturally in terms of the present age of the universe $t_{0} \sim 10^{10} \mathrm{y}[3-9]$. The same tendency is indicated also for $G[10]$. This is expected to provide an important clue to the nature of a model of unification.

The most stringent constraint ever reported is due to Shlyakhter [6,7] who exploited the exceptionally sensitive dependence of the cross section of the reaction

$$
\mathrm{n}+{ }^{149} \mathrm{Sm} \rightarrow{ }^{150} \mathrm{Sm}+\gamma
$$

on the energy of a resonance lying as low as $E_{\mathrm{r}}=97.3 \mathrm{meV}$ above threshold, which corresponds to the temperature $856^{\circ} \mathrm{C}$ and is much smaller than any of the mass scales of the strong or the electromagnetic interaction.

It was observed that the anomalous abundance of ${ }^{149} \mathrm{Sm}$ observed at Oklo $[11,12]$ can be understood rather well in terms of the reaction (11) with the fundamental-constant values observed today. An analysis of the uncertainties in the Oklo observations provides an upper bound on the possible deviation of the neutron-capture cross section $\sigma_{149}$ associated with the reaction (1) occurring 2 billions years ago from its present value. Shlyakhter concluded that $\left|\Delta \sigma_{149} / \sigma_{149}\right| \lesssim 10 \%$, which implies $\left|\Delta E_{\mathrm{r}}\right| \lesssim 20 \mathrm{meV}$. Furthermore he derived that $\left|\Delta \alpha_{\mathrm{s}} / \alpha_{\mathrm{s}}\right| \lesssim 5 \times 10^{-10} \mathrm{y}^{-1}$, or $\left|\dot{\alpha}_{s} / \alpha_{\mathrm{s}}\right| \lesssim 2.5 \times 10^{-19} \mathrm{y}^{-1}$, which represent upper bounds on the time-variability of the fundamental constant that are several orders of magnitude more stringent than any other estimate of these bounds $[3-5,8,9]$.

However, this substantial improvement of the upper-bound estimate does not seem fully appreciated, because complete details of the analysis have never been published. Another reason for the limited acceptance may be that the derivation steps are much less direct than in estimates based on quasi-stellar-object spectra [8] or on clock standards [9].

More recently Damour and Dyson [13] repeated the analysis on the same process (1) in greater detail, reaching a somewhat more conservative estimate, $-120 \mathrm{meV}<\Delta E_{\mathrm{r}}<$ $90 \mathrm{meV}$. They also showed that the result can be interpreted as an upper bound $|\dot{\alpha} / \alpha| \lesssim 6 \times$ 
$10^{-17} \mathrm{y}^{-1}$, through the time variation of the Coulomb energy which can be analyzed on a much more solid theoretical basis than for the strong-interaction coupling constant $\alpha_{\mathrm{s}}$.

Having learned, however, about the availability of the data taken even more recently, we decided to pursue the subject further. The data are samples obtained from deep underground with a geologist's expertise so as to limit potential outside contamination as much as possible. We also try to use the data from other isotopes to confirm the result obtained from Sm. Possible check of the effect of outside contamination is also planned.

We find two ranges for the allowed values of $\Delta E_{\mathrm{r}}$, one that covers the null result and another apparently indicating a nonzero effect. We try to see if we can exclude one of them by the aid of the Gd data which is admittedly subject to more uncertainties than the Sm data, due to much smaller abundance because of the more significant absorption effect. However, by taking advantage of the presence of the two low-lying isotope resonances, we are able to suggest that the range around $\Delta E_{\mathrm{r}}=0$ is favored if we accept certain plausibility arguments. A narrower constraint on the reactor temperatures obtained recently provides an added support to this conclusion.

We also show that the theoretical analysis designed originally to be applied to constrain the variability of the electromagnetic coupling constant [13] can be applied to the strong interaction as well, thanks to the exceptionally small value of $E_{\mathrm{r}}$, requiring a near cancellation between the effects of the two interactions.

We introduce basic equations that are used in our analyses: in Section 2 those related to neutron absorption and in Section 3 those related to nuclide transformations along isotope chains. New measurements on five relatively recent samples are then presented and analyzed in Section 4, providing a better upper bound on $\Delta E_{\mathrm{r}}$. Section 5 discusses the relevance of the results to the possible time-variation of the electromagnetic-interaction and the strong-interaction coupling constants. Section 6 gives discussions summary of the results obtained in our investigation. Appendix A provides some details of the numerical calculation of the thermal average of the resonance cross sections. In Appendix B we show some details of the fluence determination adopted in this investigation.

\section{Neutron absorption cross sections}

The results obtained in this investigation are based on an analysis of the neutron-absorption cross section in the reaction (11) and the corresponding reactions on Gd. The reactions are dominated by a resonance, which we describe in the standard Breit-Wigner form,

$$
\sigma_{\mathrm{r}}=\frac{g_{0} \pi \hbar^{2}}{2 m E} \frac{\Gamma_{\mathrm{n}} \Gamma_{\gamma}}{\left(E-E_{\mathrm{r}}\right)^{2}+\Gamma_{\mathrm{tot}}^{2} / 4},
$$

where $g_{0}=(2 J+1) /[(2 s+1)(2 I+1)]$ is the statistical factor, $s$ the spin of the neutron, $I$ the spin of the target, $J$ the spin of the compound nucleus, and $\Gamma_{\text {tot }}=\Gamma_{\mathrm{n}}+\Gamma_{\gamma}$ the total width in terms of the neutron and the photon widths, respectively. Also, $\Gamma_{\mathrm{n}}=(2 k / K)(D / \pi)$, where $k^{2}=2 m E / \hbar^{2}, K$ the wave number inside the target nucleus, and $D$ the level spacing of the compound levels. The values of these parameters are given in Table 1 for the lowest-lying resonance in the reactions on ${ }^{149} \mathrm{Sm},{ }^{155} \mathrm{Gd}$ and ${ }^{157} \mathrm{Gd}$. 


\begin{tabular}{||l|rrr||}
\hline Channel & ${ }^{149} \mathrm{Sm}$ & ${ }^{155} \mathrm{Gd}$ & ${ }^{157} \mathrm{Gd}$ \\
\hline Resonance energy $E_{\mathrm{r}}(\mathrm{meV})$ & 97.3 & 26.8 & 31.4 \\
Neutron width $\Gamma_{\mathrm{n}}(\mathrm{meV})$ & 0.533 & 0.104 & 0.470 \\
Gamma width $\Gamma_{\gamma}(\mathrm{meV})$ & 60.5 & 108 & 106 \\
Statistical factor $g_{0}$ & $9 / 16$ & $5 / 8$ & $5 / 8$ \\
\hline
\end{tabular}

Table 1: Parameters of the lowest-lying resonances used in the present analysis. The tabulated values of $E_{\mathrm{r}}$ are the present resonance-position values $E_{\mathrm{r} 0}$ [14]. The listed values of $\Gamma_{\mathrm{n}}$ are estimated at $E_{\mathrm{r}}$.

We now derive how the isotopic abundances observed today depend on $E_{\mathrm{r}}$, since we need to consider the possibility that $E_{\mathrm{r}}$ were different from its present value 2 billion years ago. Other parameters may be assumed constant, since only $E_{\mathrm{r}}$ will affect the result in any significant manner. Note that we would normally expect abundance changes not much larger than $\Delta \alpha / \alpha$, unless they are amplified by the resonance mechanism and by the extremely small value of $E_{\mathrm{r}}$ relative to any of the mass scales of nuclear physics.

We average (2) with respect to the thermal neutron flux,

$$
\Phi(E, T)=v(E) \rho(E, T), \quad \text { with } \quad v=\sqrt{2 E / m},
$$

where

$$
\rho(E, T)=2 \pi^{-1 / 2} T^{-3 / 2} e^{-E / T} \sqrt{E}
$$

is the normalized Maxwell-Boltzmann distribution.

Normally the thermally averaged cross section is defined by

$$
\sigma(T)=[\mathcal{D}(T)]^{-1} \int \sigma(E) \Phi(E, T) d E
$$

where

$$
\mathcal{D}(T)=\int \Phi(E, T) d E
$$

However, in most of the analyses of the Oklo phenomenon, it is customary [15] to replace the denominator $\mathcal{D}(T)$ by

$$
\hat{\mathcal{D}}=v_{0} \int \rho(E, T) d E=v_{0}
$$

for a convenient choice of the velocity $v_{0}=2200 \mathrm{~m} / \mathrm{s}$ corresponding to the temperature $T_{0}=(m / 2 k) v_{0}^{2}=20.4^{\circ} \mathrm{C}$, hence defining an effective cross section

$$
\hat{\sigma}(T)=\hat{\mathcal{D}}^{-1} \int \sigma_{\mathrm{r}}(E) \Phi(E, T) d E=\sqrt{\frac{4}{\pi} \frac{T}{T_{0}}} \sigma(T) .
$$

The advantage of this non-standard definition is that, unlike $\mathcal{D}(T), \hat{\mathcal{D}}$ is a constant, independent of $T$. We note that the neutron flux at Oklo 2 billion years ago is determined 


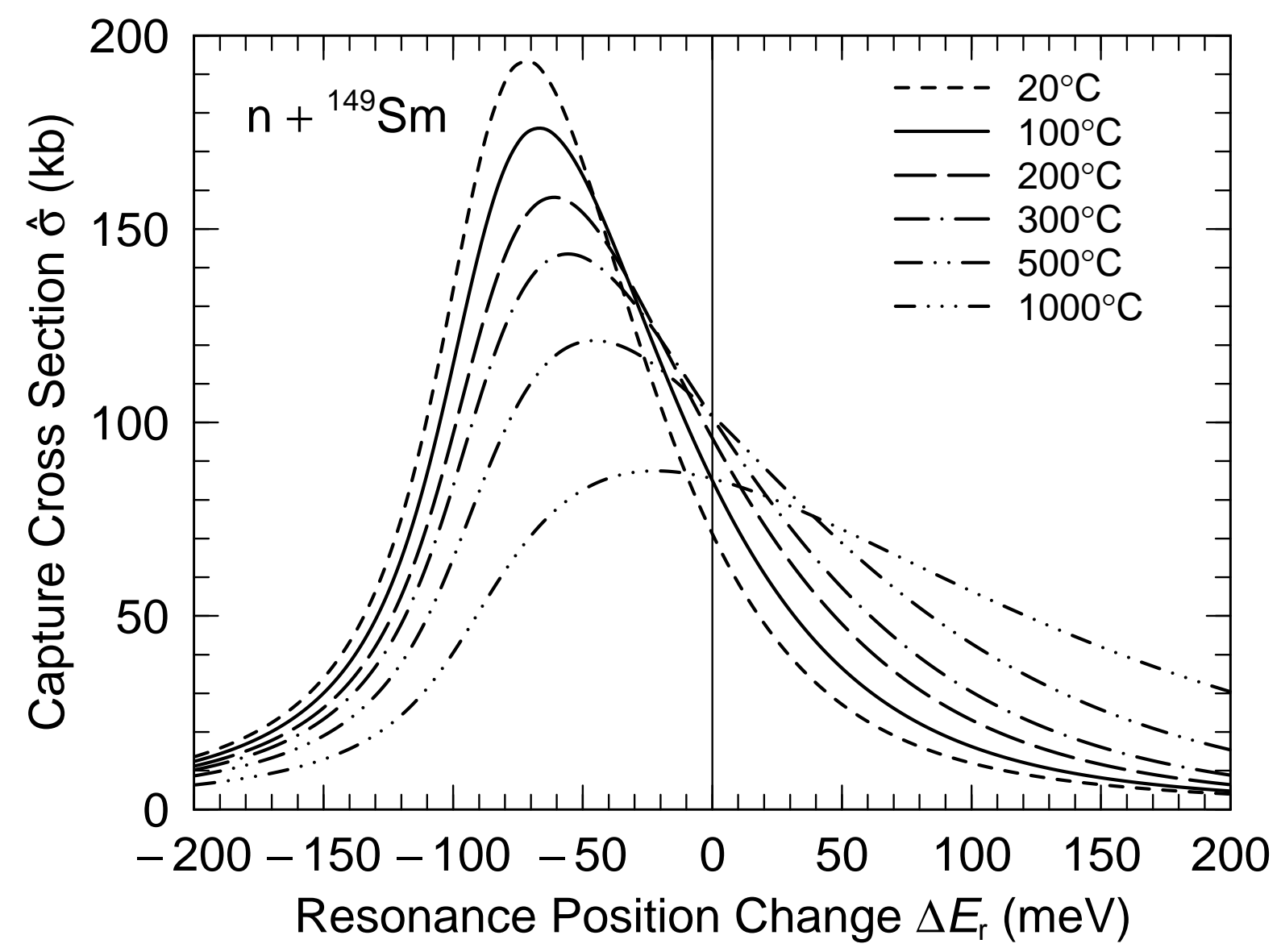

Figure 1: Calculated thermally averaged cross sections $\hat{\sigma}_{149}$ for $\mathrm{n}+{ }^{149} \mathrm{Sm} \rightarrow{ }^{150} \mathrm{Sm}+\gamma$ as functions of the resonance position change $\Delta E_{\mathrm{r}}$ and the temperature $T$.

based on the observed abundances of ${ }^{143,145} \mathrm{Nd}$ and ${ }^{147} \mathrm{Sm}$, as will be shown in more detail in Appendix B. The corresponding neutron-absorption cross sections are known to obey a $1 / v$ behavior to a good approximation, implying that the effective cross sections of these processes are nearly $T$-independent, hence simplifying the practical analysis considerably.

In the following analysis, the cross sections always occur multiplied by the thermally averaged flux $\phi(T)$. It is obviously convenient to introduce an effective flux $\hat{\phi}$ in such a way that the product with $\hat{\sigma}$ remains the same as the original product:

$$
\sigma \phi=\hat{\sigma} \hat{\phi}
$$

Using the last expression of (8), we find

$$
\hat{\phi}=\sqrt{\frac{\pi}{4} \frac{T_{0}}{T}} \phi .
$$

We evaluate the integral in (8) by numerical integration. Details of the calculation are found in Appendix A. The results for reactions on ${ }^{149} \mathrm{Sm},{ }^{155} \mathrm{Gd}$, and ${ }^{157} \mathrm{Gd}$ are shown in Figs. 1 3, respectively. The figures illustrate the strong dependence of the thermallyaveraged cross section on the resonance-position change $\Delta E_{\mathrm{r}}$ for various reactor temperatures $T$. 


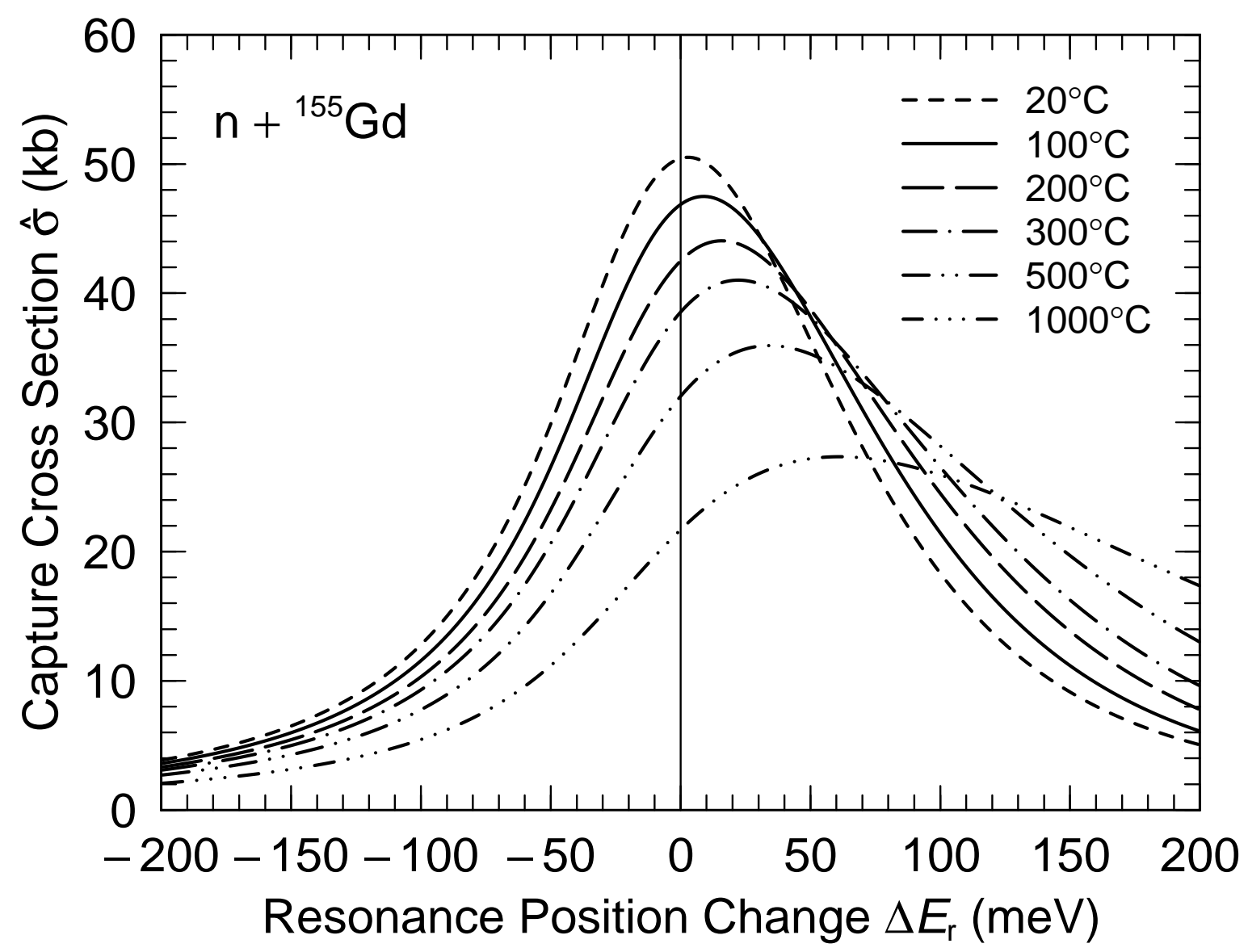

Figure 2: Calculated thermally averaged cross sections $\hat{\sigma}_{155}$ for $\mathrm{n}+{ }^{155} \mathrm{Gd} \rightarrow{ }^{156} \mathrm{Gd}+\gamma$ as functions of the resonance position change $\Delta E_{\mathrm{r}}$ and the temperature $T$.

We have calculated numerically the effect of thermal fluctuations of the atoms in the neutron absorber on the calculated cross sections. The magnitude of this so-called Doppler effect on the calculated $\hat{\sigma}(T)$ is less than $1 \%$ even at the highest temperature investigated, $1000^{\circ} \mathrm{C}$, and much smaller for lower temperatures. Therefore this effect can be safely ignored, and the formalism below is for simplicity developed without including Doppler broadening.

\section{Relations for isotopic compositions}

The isotope ratios present in Oklo today are superpositions of contributions from fission, subsequent neutron capture, and of the original natural abundances. The interpretation of the observed abundances is simplified when in special cases some processes do not contribute. In a few of those cases, where the various contributions to the now observed isotope ratios are most easily disentangled, we may draw stringent conclusions about the variation in time of a few low-lying neutron-resonance positions. We consider here the cases of neutron capture on ${ }^{149} \mathrm{Sm},{ }^{155} \mathrm{Gd}$, and ${ }^{157} \mathrm{Gd}$. 


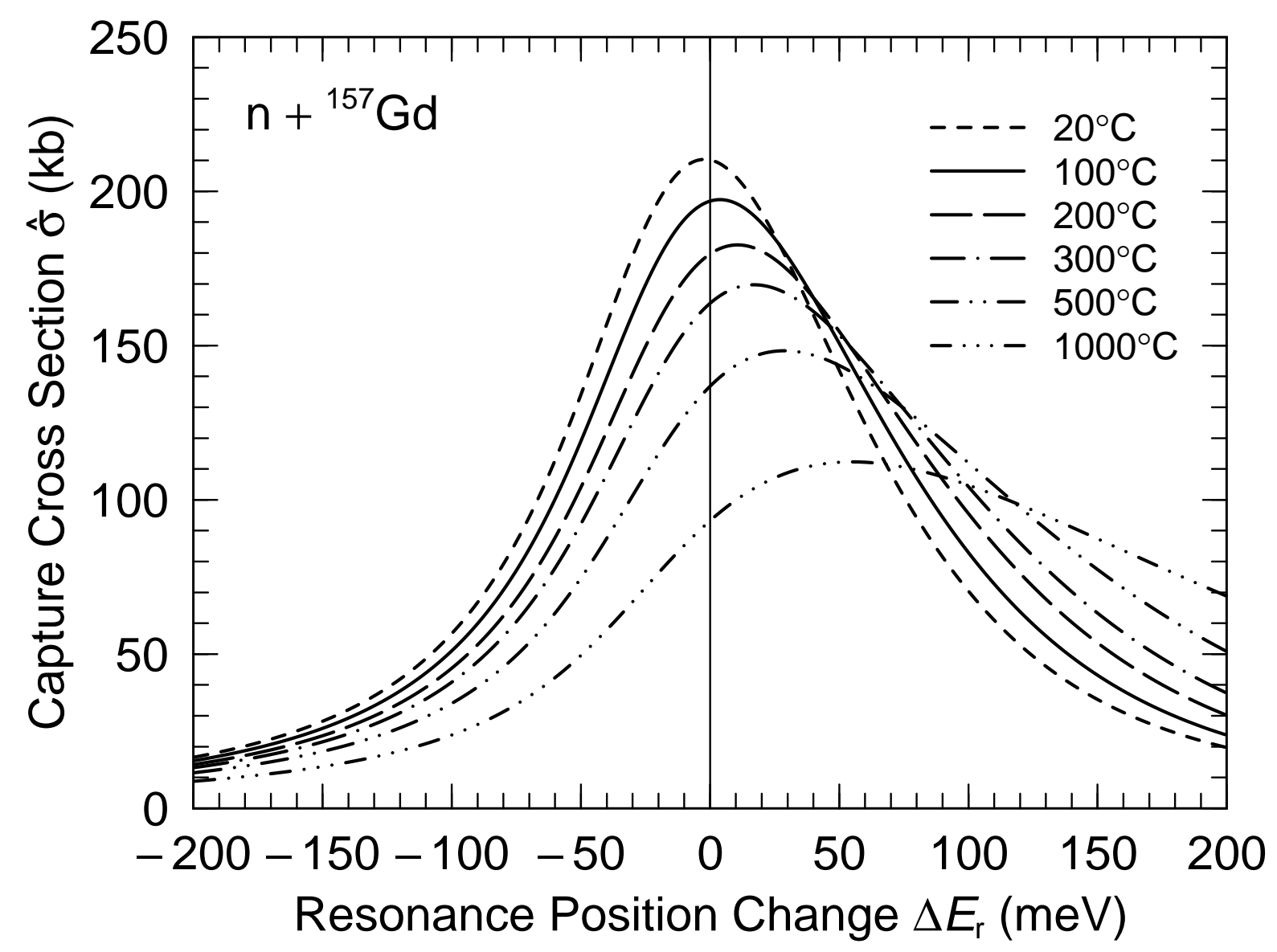

Figure 3: Calculated thermally averaged cross sections $\hat{\sigma}_{157}$ for $\mathrm{n}+{ }^{157} \mathrm{Gd} \rightarrow{ }^{158} \mathrm{Gd}+\gamma$ as functions of the resonance position change $\Delta E_{\mathrm{r}}$ and the temperature $T$.

\section{$3.1 \quad$ Isotopic composition of Sm}

We can make a few observations that simplify the differential equations obtained for the time evolution of the number of atoms per unit volume $N_{A}(t)$ of the samarium isotopes ${ }^{A} \mathrm{Sm}$ near ${ }^{149} \mathrm{Sm}$ that are connected by nuclear transformations. The spontaneous-fission half-lives of ${ }^{235} \mathrm{U}$ and ${ }^{238} \mathrm{U}$ exceed $10^{16} \mathrm{y}$. Therefore fission products are generated in appreciable amounts only by neutron-induced fission during the period of operation of the natural reactors at Oklo. Some isotopes are shielded by stable nuclides and cannot be reached in the $\beta$-decay of the unstable nuclei created during the fission process. In addition, it is possible to neglect some neutron-capture reactions because of their low cross sections, 2.413 barn [16], for example, for capture on ${ }^{148} \mathrm{Sm}$.

Thus, we obtain the following differential equations:

$$
\begin{aligned}
& \frac{d N_{147}(t)}{d t}=-\hat{\sigma}_{147} \hat{\phi} N_{147}(t)+N_{235}^{0} \exp \left(-\hat{\sigma}_{\mathrm{a}} \hat{\phi} t\right) \hat{\sigma}_{\mathrm{f} 235} \hat{\phi} Y_{147}, \\
& \frac{d N_{148}(t)}{d t}=\hat{\sigma}_{147} \hat{\phi} N_{147}(t), \\
& \frac{d N_{149}(t)}{d t}=-\hat{\sigma}_{149} \hat{\phi} N_{149}(t)+N_{235}^{0} \exp \left(-\hat{\sigma}_{\mathrm{a}} \hat{\phi} t\right) \hat{\sigma}_{\mathrm{f} 235} \hat{\phi} Y_{149},
\end{aligned}
$$


where $\hat{\sigma}_{\text {f235 }}$ is the ${ }^{235} \mathrm{U}$ fission cross section, $\hat{\sigma}_{\mathrm{a}}$ is the effective neutron-absorption cross section of ${ }^{235} \mathrm{U}$ which will be defined below, $Y_{147}$ and $Y_{149}$ are the fission yields of ${ }^{147} \mathrm{Sm}$ and ${ }^{149} \mathrm{Sm}$, respectively, and $\hat{\phi}$ the effective flux defined in Eq. (10). The effective neutronabsorption cross section $\hat{\sigma}_{\mathrm{a}}$ is not the bare fission cross section but an effective cross section which includes the restitution of ${ }^{235} \mathrm{U}$. Restitution refers to the sequence of events in which a neutron is captured by ${ }^{238} \mathrm{U}$ and ${ }^{235} \mathrm{U}$ is the final decay product created via successive $\beta$-decays yielding ${ }^{239} \mathrm{Pu}$ and a subsequent $\alpha$ decay. The way we determined the effective flux $\hat{\phi}$ and the effective neutron-absorption cross section $\hat{\sigma}_{\mathrm{a}}$ is given in Appendix $\mathrm{B}$ where these two quantities and the epi-thermal index are obtained simultaneously by solving three coupled equations. The effective neutron-absorption cross section is defined in terms of the ${ }^{235} \mathrm{U}$ total neutron-absorption cross section given by $\hat{\sigma}_{235}$ as

$$
\hat{\sigma}_{\mathrm{a}}=\left(1-C_{\mathrm{rz}}\right) \hat{\sigma}_{235}
$$

The interpretation of this definition is clear when we refer the third of the four equations just above equation (B.12). The time $t$ starts at the beginning of the reactor period, with $\hat{\phi}$ assumed to be constant in time. Obviously $\hat{\sigma}_{149}$ is dominated by the thermally averaged cross section of the absorption process (11) while $\hat{\sigma}_{147}$ is the non-resonant absorption cross section of ${ }^{147} \mathrm{Sm}$.

The initial number densities $N_{A}(0)$ are $N_{235}^{0}$ for ${ }^{235} \mathrm{U}$ and

$$
N_{A}(0)=N^{\text {nat }} R_{A}^{\text {nat }}
$$

for the samarium isotopes, for which the relative fractional natural abundances $R_{A}^{\text {nat }}$ have been observed to be [16]:

$$
R_{144}^{\text {nat }}=0.0310, \quad R_{147}^{\text {nat }}=0.1500, \quad R_{148}^{\text {nat }}=0.1130, \quad \text { and } \quad R_{149}^{\text {nat }}=0.1380 .
$$

The value for the stable and inactive ${ }^{144} \mathrm{Sm}$ is included, because it is needed later, whereas the final results will be independent of the overall normalization constant $N^{\text {nat }}$ and the capture cross section $\hat{\sigma}_{147}$.

The solution of the system of differential equations (11) is:

$$
\begin{aligned}
& N_{149}(t)=\frac{N_{235}^{0} \hat{\sigma}_{\mathrm{f} 235} Y_{149}}{\hat{\sigma}_{\mathrm{a}}-\hat{\sigma}_{149}}\left[\exp \left(-\hat{\sigma}_{149} \hat{\phi} t\right)-\exp \left(-\hat{\sigma}_{\mathrm{a}} \hat{\phi} t\right)\right]+N^{\mathrm{nat}} R_{149}^{\mathrm{nat}} \exp \left(-\hat{\sigma}_{\mathrm{a}} \hat{\phi} t\right), \\
& N_{147}(t)+N_{148}(t)=\frac{N_{235}^{0} Y_{147} \hat{\sigma}_{\mathrm{f} 235}}{\hat{\sigma}_{\mathrm{a}}}\left[1-\exp \left(-\hat{\sigma}_{\mathrm{a}} \hat{\phi} t\right)\right]+N^{\mathrm{nat}}\left(R_{147}^{\mathrm{nat}}+R_{148}^{\mathrm{nat}}\right),
\end{aligned}
$$

from which it follows that

$$
\begin{aligned}
& \frac{N_{147}\left(t_{1}\right)+N_{148}\left(t_{1}\right)}{N_{149}\left(t_{1}\right)}= \\
& \frac{\frac{\hat{\sigma}_{\mathrm{f} 235} k Y_{147}}{\hat{\sigma}_{\mathrm{a}}}\left[1-\exp \left(-\hat{\sigma}_{\mathrm{a}} \hat{\phi} t_{1}\right)\right]+\left(R_{147}^{\text {nat }}+R_{148}^{\mathrm{nat}}\right)}{\frac{k \hat{\sigma}_{\mathrm{f} 235} Y_{149}}{\hat{\sigma}_{\mathrm{a}}-\hat{\sigma}_{149}}\left[\exp \left(-\hat{\sigma}_{149} \hat{\phi} t_{1}\right)-\exp \left(-\hat{\sigma}_{\mathrm{a}} \hat{\phi} t_{1}\right)\right]+R_{149}^{\text {nat }} \exp \left(-\hat{\sigma}_{\mathrm{a}} \hat{\phi} t_{1}\right)},
\end{aligned}
$$

\footnotetext{
${ }^{\dagger}$ Throughout this work, we used this assumption. However, if we use the technique used in Ref. [13], it is possible to remove this assumption. Then $\hat{\phi} t$ should be replaced $\int \hat{\phi} d t$ in all our expressions. The numerical results given later are unchanged even if we use this generalized expression.
} 
at $t_{1}$, the end of the reactor activity. Since then the samarium isotope concentrations $N_{A}(t)$ have remained unchanged until today, unless corresponding, naturally-occurring isotopes have flowed into the sample regions from the outside.

The ratio $k \equiv N_{235}^{0} / N^{\text {nat }}$ can be determined in the following way. We observe that ${ }^{144} \mathrm{Sm}$ is never produced as a fission product and has a negligible capture cross section, 1.64 barn [16], for neutron capture, that is $N_{144}(t)$ is constant. We may then calculate the ratio $N_{144}\left(t_{1}\right) /\left(N_{147}\left(t_{1}\right)+N_{148}\left(t_{1}\right)\right)$ and compare to the observed ratio:

$$
\begin{aligned}
& {\left[\frac{N_{144}\left(t_{1}\right)}{N_{147}\left(t_{1}\right)+N_{148}\left(t_{1}\right)}\right]_{\text {Observed }} \equiv R_{\mathrm{O}}} \\
& {\left[\frac{N_{144}\left(t_{1}\right)}{N_{147}\left(t_{1}\right)+N_{148}\left(t_{1}\right)}\right]_{\text {Calculated }}=} \\
& \frac{N^{\text {nat }} R_{144}^{\text {nat }}}{N_{235}^{0} Y_{147} \frac{\hat{\sigma}_{\text {f235 }}}{\hat{\sigma}_{\mathrm{a}}}\left[1-\exp \left(-\hat{\sigma}_{\mathrm{a}} \phi t_{1}\right)\right]+N^{\text {nat }}\left(R_{147}^{\text {nat }}+R_{148}^{\text {nat }}\right)}= \\
& \frac{R_{144}^{\text {nat }}}{k Y_{147} \frac{\hat{\sigma}_{\text {f235 }}\left[1-\exp \left(-\hat{\sigma}_{\mathrm{a}} \phi t_{1}\right)\right]+\left(R_{147}^{\text {nat }}+R_{148}^{\text {nat }}\right)}{\hat{\sigma}_{\mathrm{a}}} .}
\end{aligned}
$$

From these equations, we obtain

$$
k=\frac{R_{144}^{\mathrm{nat}}-R_{\mathrm{O}}\left(R_{147}^{\mathrm{nat}}+R_{148}^{\mathrm{nat}}\right)}{R_{\mathrm{O}} Y_{147} \frac{\hat{\sigma}_{\mathrm{f} 235}}{\hat{\sigma}_{\mathrm{a}}}\left[1-\exp \left(-\hat{\sigma}_{\mathrm{a}} \phi t_{1}\right)\right]} .
$$

which can be estimated from the observed values only.

We finally solve (16) for $\hat{\sigma}_{149}$ by using observed abundances, and the fluence $\hat{\phi} t_{1}$ and the effective neutron-absorption cross section $\hat{\sigma}_{\mathrm{a}}$ defined earlier and discussed in Appendix B. The values of fluences and effective cross sections are tabulated in Ref. [17] and using them, we obtain the results for five recently analyzed samples shown in Table 2 .

\subsection{Isotopic composition of $\mathrm{Gd}$}

For gadolinium we may define relative fractional natural abundances $R_{A}^{\text {nat }}$ in complete analogy with samarium in Eq. (13). For Gd the relative fractional natural abundances for the relevant isotopes are

$$
\begin{array}{ll}
R_{155}^{\text {nat }}=0.1480, & R_{156}^{\text {nat }}=0.2047, \quad R_{157}^{\text {nat }}=0.1565, \\
R_{158}^{\text {nat }}=0.2484, & \text { and } \quad R_{160}^{\text {nat }}=0.2186 .
\end{array}
$$

Because thermal neutron capture cross section of ${ }^{160} \mathrm{Gd}$ is 0.796 barn [16], we can use the value of the nearly constant $N_{160}(t)$ observed at each sample location to determine $N^{\text {nat }}$. 


\begin{tabular}{||l|rrrrr||}
\hline & \multicolumn{5}{|c||}{ Sample } \\
& SF84-1469 & SF84-1480 & SF84-1485 & SF84-1492 & SD.37 \\
\hline$\hat{\phi} t_{1}(1 / \mathrm{kb})$ & 0.525 & 0.798 & 0.622 & 0.564 & 0.780 \\
$N_{144}\left(t_{1}\right)(\%)$ & 0.1052 & 0.2401 & 0.2073 & 0.1619 & 0.06909 \\
$N_{147}\left(t_{1}\right)(\%)$ & 55.34 & 53.23 & 54.03 & 54.81 & 52.74 \\
$N_{148}\left(t_{1}\right)(\%)$ & 2.796 & 3.468 & 3.079 & 2.890 & 4.694 \\
$N_{149}\left(t_{1}\right)(\%)$ & 0.5544 & 0.2821 & 0.4466 & 0.4296 & 0.3088 \\
$N_{235}\left(t_{1}\right) / N_{238}\left(t_{1}\right)$ & 0.03181 & 0.02665 & 0.02971 & 0.03047 & 0.02435 \\
\hline$\hat{\sigma}_{149}(\mathrm{~kb})$ & 85.6 & 96.5 & 83.8 & 99.0 & 89.5 \\
\hline
\end{tabular}

Table 2: Measured isotopic ratios and fluences for Sm for five samples and corresponding, calculated values of the cross section $\hat{\sigma}_{149}$.

Because the neutron-absorption cross sections $\hat{\sigma}_{156}$ and $\hat{\sigma}_{158}$ are 2.188 barn and 2.496 barn [16], respectively, and because ${ }^{154} \mathrm{Gd}$ is shielded by the stable ${ }^{154} \mathrm{Sm}$ the study of the possible shifts of the low-lying resonances in ${ }^{155} \mathrm{Gd}$ and ${ }^{157} \mathrm{Gd}$ is considerably simplified: we may study the transmutations related to the isotope pairs ${ }^{155} \mathrm{Gd}-{ }^{156} \mathrm{Gd}$ and ${ }^{157} \mathrm{Gd}-{ }^{158} \mathrm{Gd}$ separately. The differential equations for the number of atoms $N_{A}(t)$ per unit volume for the first isotope pair are:

$$
\begin{aligned}
& \frac{d N_{155}(t)}{d t}=-\hat{\sigma}_{155} \hat{\phi} N_{155}(t)+N_{235}^{0} \exp \left(-\hat{\sigma}_{\mathrm{a}} \hat{\phi} t\right) \hat{\sigma}_{\mathrm{f} 235} \hat{\phi} Y_{155}, \\
& \frac{d N_{156}(t)}{d t}=+\hat{\sigma}_{155} \hat{\phi} N_{155}(t)+N_{235}^{0} \exp \left(-\hat{\sigma}_{\mathrm{a}} \hat{\phi} t\right) \hat{\sigma}_{\mathrm{f} 235} \hat{\phi} Y_{156},
\end{aligned}
$$

where we ignored the small cross section $\hat{\sigma}_{156}=2.188$ barn. We find the solution:

$$
\begin{aligned}
N_{155}(t)= & \frac{N_{235}^{0} \hat{\sigma}_{f 235} Y_{155}}{\hat{\sigma}_{\mathrm{a}}-\hat{\sigma}_{155}}\left[\exp \left(-\hat{\sigma}_{155} \hat{\phi} t\right)-\exp \left(-\hat{\sigma}_{\mathrm{a}} \hat{\phi} t\right)\right] \\
& +N^{\text {nat }} R_{155}^{\text {nat }} \exp \left(-\hat{\sigma}_{155} \hat{\phi} t\right), \\
N_{155}(t)+N_{156}(t)= & N_{235}^{0}\left(Y_{155}+Y_{156}\right) \frac{\hat{\sigma}_{\text {2235 }}}{\hat{\sigma}_{\mathrm{a}}}\left[1-\exp \left(-\hat{\sigma}_{\mathrm{a}} \hat{\phi} t\right)\right] \\
& +N^{\text {nat }}\left(R_{155}^{\text {nat }}+R_{156}^{\text {nat }}\right),
\end{aligned}
$$

In analogy with Eq. (17) we introduce

$$
R_{\mathrm{O}}=\frac{N_{160}\left(t_{1}\right)}{N_{155}\left(t_{1}\right)+N_{156}\left(t_{1}\right)}
$$

which can be compared with the observed data expressed in terms of $N_{160} / N_{156}$ and $N_{155} / N_{156}$ with reference to the stable isotope ${ }^{160} \mathrm{Gd}$. In analogy with Eq. (18) the ratio 
$k \equiv N_{235}^{0} / N^{\text {nat }}$ is here given by

$$
k=\frac{R_{160}^{\text {nat }}-R_{0}\left(R_{155}^{\text {nat }}+R_{156}^{\text {nat }}\right)}{R_{\mathrm{O}} \frac{\hat{\sigma}_{\mathrm{f} 235}}{\hat{\sigma}_{\mathrm{a}}}\left(Y_{155}+Y_{156}\right)\left[1-\exp \left(-\hat{\sigma}_{\mathrm{a}} \hat{\phi} t_{1}\right)\right]} .
$$

Now $\hat{\sigma}_{155}$ can be determined by solving iteratively

$$
\frac{N_{155}\left(t_{1}\right)}{N_{156}\left(t_{1}\right)}=\frac{A}{B}
$$

where the observed ratio is substituted into the left-hand side, and where

$$
\begin{aligned}
A & =\frac{k \hat{\sigma}_{\mathrm{f} 235} Y_{155}}{\hat{\sigma}_{\mathrm{a}}-\hat{\sigma}_{155}}\left[\exp \left(-\hat{\sigma}_{155} \hat{\phi} t_{1}\right)-\exp \left(-\hat{\sigma}_{\mathrm{a}} \hat{\phi} t_{1}\right)\right]+R_{155}^{\mathrm{nat}} \exp \left(-\hat{\sigma}_{155} \hat{\phi} t_{1}\right), \\
B & =\frac{k Y_{155}}{\hat{\sigma}_{155}-\hat{\sigma}_{\mathrm{a}}} \frac{\hat{\sigma}_{\mathrm{f} 235}}{\hat{\sigma}_{\mathrm{a}}}\left(\hat{\sigma}_{155}\left[1-\exp \left(-\hat{\sigma}_{\mathrm{a}} \hat{\phi} t_{1}\right)\right]-\hat{\sigma}_{\mathrm{a}}\left[1-\exp \left(-\hat{\sigma}_{155} \hat{\phi} t_{1}\right)\right]\right) \\
& +k Y_{156} \frac{\hat{\sigma}_{\mathrm{f} 235}}{\hat{\sigma}_{\mathrm{a}}}\left[1-\exp \left(-\hat{\sigma}_{\mathrm{a}} \hat{\phi} t_{1}\right)\right]+R_{155}^{\mathrm{nat}}\left[1-\exp \left(-\hat{\sigma}_{155} \hat{\phi} t_{1}\right)\right]+R_{156}^{\mathrm{nat}} .
\end{aligned}
$$

The transmutation equations related to the isotope pairs ${ }^{157} \mathrm{Gd}$ and ${ }^{158} \mathrm{Gd}$ are obtained by replacing quantities related to ${ }^{155} \mathrm{Gd}$ and ${ }^{156} \mathrm{Gd}$ by quantities related to ${ }^{157} \mathrm{Gd}$ and ${ }^{158} \mathrm{Gd}$, respectively, in the above equations.

\section{Analysis of the data}

We use the isotopic data of five samples recently taken from reactor zones (hereafter called RZs) 10 and 13 which are located deep underground, 150-250 m below the surface, while the other RZs 1-9 are located near the surface. The use of samples from deep-lying RZs is important for this study, because it minimizes contamination from in-flow of isotopes from the outside after the cessation of reactor operation. Particularly relevant factors that influence the contamination process are:

1. Hexavalent uranium U(VI) species are water-soluble, while tetravalent species are insoluble. The valence of uranium is largely determined by oxidation conditions in nature.

2. A uraninite sample located near the surface easily forms water-soluble species because of contact with the oxidizing atmosphere. On the other hand, a sample deep underground remains insoluble because of a relatively reducing environment.

3. Uranium minerals under oxidizing conditions are dissolved and recrystalized. It is possible that rare-earth elements originally incorporated into uranium minerals are released as the minerals are dissolved and precipitated on rock surfaces.

It is obvious that any geological alteration of the RZs would reduce the quality of the isotopic data. Rare-earth-element enriched regions were actually found in layers around RZs 1 to 9 [18,19]. For example, the Sm content around GL13 at RZ 9 shows a variation 
in the range from 21 to $285 \mathrm{ppm}$, a marked difference from the range 0.43 to $3.32 \mathrm{ppm}$ observed in SF84 which is close to RZ 10 used in this study. This geological observation suggests that we as a precautionary measure, to keep the contamination to a minimum, collect samples from deep-lying RZs. The quality of our new data obtained in this way is, for example, demonstrated by a much smaller scatter of the values we obtained for $\hat{\sigma}_{149}$ compared to what was obtained in Ref. [13] from earlier samples taken from RZs 1-9.

\subsection{Description of the samples}

Because near the surface, rare-earth elements and $U$ often fail to form closed systems, they are likely get mixed with outside, normal isotope distributions. This may yield an estimate of the fluence that is either too large or too small. We selected our samples in order to minimize the effect due to this process. Four samples from SF84 were taken from a single drill core which crosscuts RZ 10 located $150 \mathrm{~m}$ deeper than the RZs near the surface. The SD.37 sample was from a part which was highly depleted in ${ }^{235} \mathrm{U}$, in a borehole which crosscuts RZ 13. Detailed characterization of the reactor was performed through chemical and isotopic analyses of fission products and $U$ taken every few $\mathrm{cm}$ from the drill core [17].

\subsection{Sm data}

Table 2 shows measured and calculated results for the five samples labeled in the first row. The first four samples were taken from RZ 10, whereas the last sample was taken from RZ 13. Measurement errors are given in Ref. [17]. Because they are mostly below the level of percent they are negligible in our analysis. More details on this question will be reported elsewhere. The last row shows $\hat{\sigma}_{149}$ calculated as described in Subsection 3.1. The result can be represented by

$$
\hat{\sigma}_{149}=91 \pm 6 \mathrm{~kb}
$$

We then use Fig. 1 to estimate $\Delta E_{\mathrm{r}}=E_{\mathrm{r}}-E_{\mathrm{r} 0}$, for the assumed temperatures, with the results shown in Fig. 目.

Note that for the temperature range of interest there are two separated regions for the allowed values $\Delta E_{\mathrm{r}}$ for a given set of $\hat{\sigma}_{149}$ and the lower and the upper temperature limits $T_{\text {lower }}$ and $T_{\text {upper. }}$. In Fig. 1, these regions are bounded by both ends of (26), $T_{\text {lower }}$ and $T_{\text {upper }}$, and the left-branch of the curves for one while the right-branch for the other. The right-branch range passes through the area that covers $\Delta E_{\mathrm{r}}=0$ whereas the left-branch range extends to the far-negative region of $\Delta E_{\mathrm{r}}$.

By taking advantage of the strong temperature-dependence of the neutron-capture cross section of ${ }^{176} \mathrm{Lu}$, most estimates of the reactor temperature have been made based on observed isotopic ratios of ${ }^{176} \mathrm{Lu} /{ }^{175} \mathrm{Lu}$. It has been suspected, however, that the result is highly problematic. In one of the careful analyses the temperature range $200-360^{\circ} \mathrm{C}$ was obtained [20], whereas the same technique applied to another set of samples produced $380^{\circ} \mathrm{C}$ for one sample with all the six others yielding $>1000^{\circ} \mathrm{C}$ [17], indicating still unknown flaws, such as the lack of more precisely determined temperature-dependence of the 


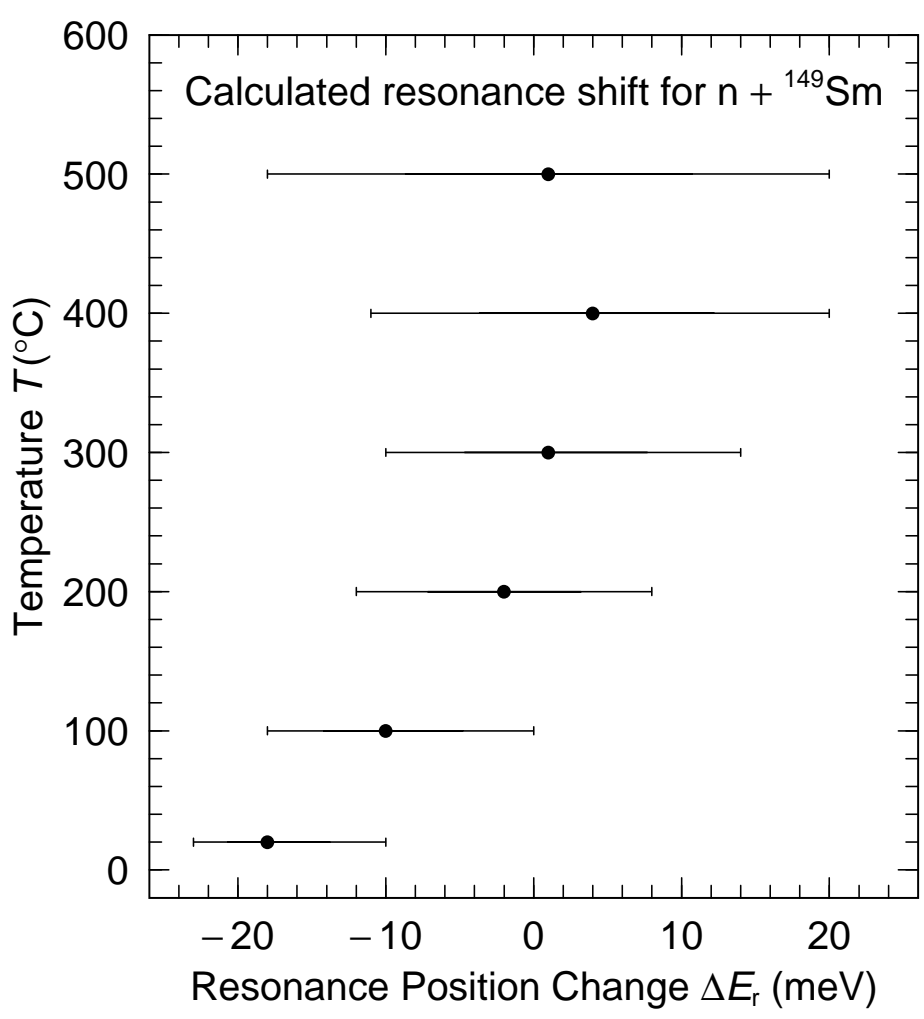

Figure 4: The ranges of $\Delta E_{\mathrm{r}}$, represented by horizontal lines, calculated from Fig. 1 corresponding to the estimated cross section $\hat{\sigma}_{149}$ given by (25) are shown for several choices of the temperature, namely $T=20,100,200,300,400,500^{\circ} \mathrm{C}$.

capture cross section particularly at higher temperatures, possible outside contamination, and others, depending on different conditions of reactors.

In view of this complicated and even confusing situation, it might be worth looking for other types of approach. A range of $300-350^{\circ} \mathrm{C}$ was obtained, for example, by measuring the oxygen isotopes of chlorites [21] outside reactor cores, though less direct. This is supported by the most recent analysis by Pourcelot and Gauthier-Lafaye who have obtained the reactor temperature from the crystallization temperature of chlorites in the reactors [22]. Based on this result, and by considering other possible factors, like distance to the reactor core and the cooling efficiency, the authors concluded $200-400^{\circ} \mathrm{C}$, which we adopt here as the most reliable temperature range currently available. Notice that this range is consistent with the Lu result in Ref. [20].

We then obtain

$$
\Delta E_{\mathrm{r}}=4 \pm 16 \mathrm{meV}, \quad \text { for } \quad T=200-400^{\circ} \mathrm{C}
$$

for the right-branch range, while

$$
\Delta E_{\mathrm{r}}=-97 \pm 8 \mathrm{meV}, \quad \text { for } \quad T=200-400{ }^{\circ} \mathrm{C},
$$

for the left-branch range. The standard deviation has been determined from the lower and upper limits of the temperature. 


\begin{tabular}{||l|rrrrr||}
\hline$\zeta$ & SF84-1469 & SF84-1480 & SF84-1485 & SF84-1492 & SD.37 \\
\hline 0.00 & 85.6 & 96.5 & 83.8 & 99.0 & 89.5 \\
0.01 & 86.4 & 100.2 & 85.6 & 100.7 & 90.4 \\
0.02 & 87.1 & 104.3 & 87.4 & 102.4 & 91.3 \\
0.03 & 87.8 & 108.6 & 89.3 & 104.2 & 92.2 \\
0.04 & 88.6 & 113.4 & 91.2 & 106.0 & 93.2 \\
\hline
\end{tabular}

Table 3: Estimated cross sections $\hat{\sigma}_{149}(\mathrm{~kb})$ for five values of the post-reactor contamination $\zeta$ for five samples.

We also notice that restricting the temperature range further to $300-400{ }^{\circ} \mathrm{C}$, for examples, as suggested by Refs. [20,22], does not improve the result (27) in any significant manner;

$$
\Delta E_{\mathrm{r}}=5 \pm 16 \mathrm{meV}, \text { for } T=300-400^{\circ} \mathrm{C} .
$$

We point out that the shift as large as $\sim-100 \mathrm{meV}$ suggested in (28) would imply a level even below the threshold, hence requiring a more careful analysis. It seems still unlikely that it would change substantially the claim that $E_{\mathrm{r}}$ at the time of the natural reactor was different from the present value beyond many standard deviations.

The bound thus obtained for $\Delta E_{\mathrm{r}}$, particularly for the right-branch, are rather insensitive to an increase of $T_{\text {upper }}$ as long as $T_{\text {upper }} \lesssim T_{\text {crit }} \approx 820^{\circ} \mathrm{C}$, a critical temperature above which the two regions are bridged to each other. In fact for $T_{\text {upper }}=700^{\circ} \mathrm{C}$, for example, we find $-83 \pm 22 \mathrm{meV}$, and $11 \pm 10 \mathrm{meV}$ for the left-branch and the right-branch ranges, respectively. The separation between the centers of the two ranges is $94 \mathrm{meV}$, which is nearly three times as large compared with the sum $32 \mathrm{meV}$ of the half-widths of each region. Even if we take the two standard deviation bound for $\hat{\sigma}_{149}$, the critical temperature is estimated to be $T_{\text {crit }} \approx 730^{\circ} \mathrm{C}$, and the ranges for $T_{\text {upper }}=700^{\circ} \mathrm{C}$ are found to be $-78 \pm 30 \mathrm{meV}$ and $6 \pm 26 \mathrm{meV}$, respectively, which are still well separated, with the separation $84 \mathrm{meV}$ and the sum of the half-widths $56 \mathrm{meV}$.

It seems also useful to note that assuming temperatures $\gtrsim 1000^{\circ} \mathrm{C}$ leaves little room for $\Delta E_{\mathrm{r}}$ in Fig. 1 for (26), making higher temperatures less likely from the point of view of theoretical analyses.

As we alluded to before, one may suspect that the samples have been subjected to some post-reactor contamination. To estimate this effect, we assume that a fraction $\zeta$ of the natural abundance $N^{\text {nat }}$ (see (14)) flowed into the reactor from the outside environment, contributing to the observed amount of Sm. We subtract this inflow from the now observed isotope abundances and apply the preceding analysis to the remainder to re-calculate $\hat{\sigma}_{149}$. As we can see in Table 3, the result is quite insensitive to $\zeta$ as long as it stays around a few percent. This contamination range is suggested by our analysis of Gd below.

For $\zeta=0.04$, for example, we obtain

$$
\hat{\sigma}_{149}=99 \pm 10 \mathrm{~kb}
$$




\begin{tabular}{||l|rrrrr||}
\hline & \multicolumn{7}{|c||}{ Sample } \\
& SF84-1469 & SF84-1480 & SF84-1485 & SF84-1492 & SD.37 \\
& & & & & \\
\hline$N_{155}\left(t_{1}\right)(\%)$ & 0.5006 & 0.4608 & 0.6065 & 0.3899 & 0.5915 \\
$N_{156}\left(t_{1}\right)(\%)$ & 30.03 & 29.46 & 30.79 & 29.90 & 30.37 \\
$N_{157}\left(t_{1}\right)(\%)$ & 0.0418 & 0.2641 & 0.1921 & 0.0505 & 0.1881 \\
$N_{158}\left(t_{1}\right)(\%)$ & 23.66 & 25.07 & 26.52 & 23.68 & 17.31 \\
$N_{160}\left(t_{1}\right)(\%)$ & 11.21 & 12.24 & 12.93 & 10.95 & 6.1030 \\
\hline$\hat{\sigma}_{155}(\mathrm{~kb})$ & 30.9 & 16.8 & 17.8 & 36.7 & 26.3 \\
$\hat{\sigma}_{157}(\mathrm{~kb})$ & 83.3 & 8.0 & 14.3 & 73.7 & 23.3 \\
\hline
\end{tabular}

Table 4: Measured isotopic rations for Gd for five samples and the corresponding, calculated cross sections $\hat{\sigma}_{155}$ and $\hat{\sigma}_{157}$.

instead of (26), from which follows

$$
\Delta E_{\mathrm{r}}=2 \pm 14 \mathrm{meV}
$$

for either of the temperature ranges $200-400^{\circ} \mathrm{C}$ and $300-400^{\circ} \mathrm{C}$. Although the result (30) seems worse than (26), the result (31) for $\Delta E_{\mathrm{r}}$ puts an even stronger limit on its timevariation than (27), because the curves corresponding to different temperatures in Fig. 11 happen to all intersect near $\Delta E_{\mathrm{r}}=0$.

\subsection{Gd data}

In analogy with our studies of the Sm isotopic compositions and estimates of neutroncapture cross sections tabulated in Table 2 we now study the measured Gd isotopic compositions in the Oklo RZs, which are tabulated in Table 4 . To obtain this table, we used the fluence and effective cross-section values which are tabulated in [17] in the same way as we did for Sm case. By use of the formalism developed in Section 3.2 we obtain the estimates for the neutron-capture cross sections listed in the bottom two lines of Table 4. We find that the resonance-position change $\Delta E_{\mathrm{r}}$ deduced from the cross sections in Table 1 are generally significantly different from zero in contrast to what was indicated by the Sm results. It is therefore natural to investigate if the effect of contamination here, for $\mathrm{Gd}$, plays a more crucial role than for Sm, which we found was relatively insensitive to modest contaminations. For Gd we divide the five samples into two different classes, which are presented separately in Tables 5 and 6. The samples SF84-1469 and SF84-1492 presented in Table 5 are characterized by much smaller abundances of ${ }^{157} \mathrm{Gd}$ than are the other three samples presented in Table 6 .

We assume that for each sample the unknown contamination parameter $\zeta$ is the same for ${ }^{155} \mathrm{Gd}$ and ${ }^{157} \mathrm{Gd}$, and investigate if there is any $\zeta$ for which we obtain the same $\Delta E_{\mathrm{r}}$ for both isotopes. This is based on the assumption that the mass scale $\mathcal{M}$ that relates 


\begin{tabular}{|c|c|c|c|c|}
\hline \multirow[b]{2}{*}{$\zeta$} & \multicolumn{2}{|c|}{ SF84-1469 } & \multicolumn{2}{|c|}{ SF84-1492 } \\
\hline & $\begin{array}{l}\hat{\sigma}_{155} \\
(\mathrm{~kb})\end{array}$ & $\begin{array}{l}\hat{\sigma}_{157} \\
(\mathrm{~kb})\end{array}$ & $\begin{array}{l}\hat{\sigma}_{155} \\
(\mathrm{~kb})\end{array}$ & $\begin{array}{l}\hat{\sigma}_{157} \\
(\mathrm{~kb})\end{array}$ \\
\hline 0.000 & 30.9 & 83.3 & 36.7 & 73.7 \\
\hline 0.001 & 31.4 & 102.9 & 37.4 & 87.2 \\
\hline 0.002 & 31.9 & 134.7 & 38.1 & 106.6 \\
\hline 0.003 & 32.3 & 195.0 & 38.8 & 137.1 \\
\hline
\end{tabular}

Table 5: Calculated cross sections $\hat{\sigma}_{155}$ and $\hat{\sigma}_{157}$ for two samples and four values of the postreactor contamination $\zeta$.

$\Delta E_{\mathrm{r}}$ to $\Delta \alpha$ are the same for the two isotopes. In fact $\mathcal{M}$ defined by (see (34) later)

$$
\alpha \frac{d \Delta E_{\mathrm{r}}}{d \alpha}=\mathcal{M},
$$

is found to be nearly common $(\sim-1.1 \mathrm{MeV})$ according to a phenomenological BetheWeizsäcker formula, for example. Although the result might be corrected roughly by a factor of two if a more precise measurement is used [13], we consider the above simple estimate to be sufficient for our present purpose. In the same context, it seems reasonable to assume that $\mathcal{M}$ is nearly the same also for $\mathrm{Sm}$, at least sharing the same sign.

For the sample SD.37 we have in Fig. 5 plotted $\Delta E_{\mathrm{r}}(\zeta)$ determined in the following way. The neutron-capture cross sections $\hat{\sigma}_{155}$ and $\hat{\sigma}_{157}$ corresponding to the observed isotopic distributions with the assumed contamination removed, are calculated. The resonanceposition changes corresponding to these cross sections are then obtained for the assumed reactor temperature limits from the appropriate curves in Figs. 2 and 3 .

Like in Fig. 1, we have also two ranges. It is now the left-branch ranges that passes

\begin{tabular}{||c|rrrrrr||}
\hline \multirow{2}{*}{$\zeta$} & \multicolumn{2}{|c}{ SF84-1480 } & \multicolumn{2}{c|}{ SF84-1485 } & \multicolumn{2}{c||}{ SD.37 } \\
& $\begin{array}{r}\hat{\sigma}_{155} \\
(\mathrm{~kb})\end{array}$ & $\hat{\sigma}_{157}(\mathrm{~kb})$ & $\begin{array}{l}\hat{\sigma}_{155} \\
(\mathrm{~kb})\end{array}$ & $\begin{array}{r}\hat{\sigma}_{157} \\
(\mathrm{~kb})\end{array}$ & $\begin{array}{r}\hat{\sigma}_{155} \\
(\mathrm{~kb})\end{array}$ & $\begin{array}{r}\hat{\sigma}_{157} \\
(\mathrm{~kb})\end{array}$ \\
\hline 0.00 & 16.8 & 8.0 & 17.8 & 14.3 & 26.3 & 23.3 \\
0.01 & 20.2 & 11.1 & 20.6 & 26.4 & 28.1 & 29.9 \\
0.02 & 25.4 & 20.6 & 24.4 & 236.9 & 30.2 & 42.0 \\
0.03 & 34.4 & 227.1 & 30.1 & - & 32.7 & 70.9 \\
0.04 & 53.5 & - & 39.2 & - & 35.6 & 235.3 \\
\hline
\end{tabular}

Table 6: Calculated cross sections $\hat{\sigma}_{155}$ and $\hat{\sigma}_{157}$ for three samples and five values of the postreactor contamination $\zeta$. Dash $(-)$ entries are given when the calculated cross sections are much larger than any of those occurring in Fig. 3 . 


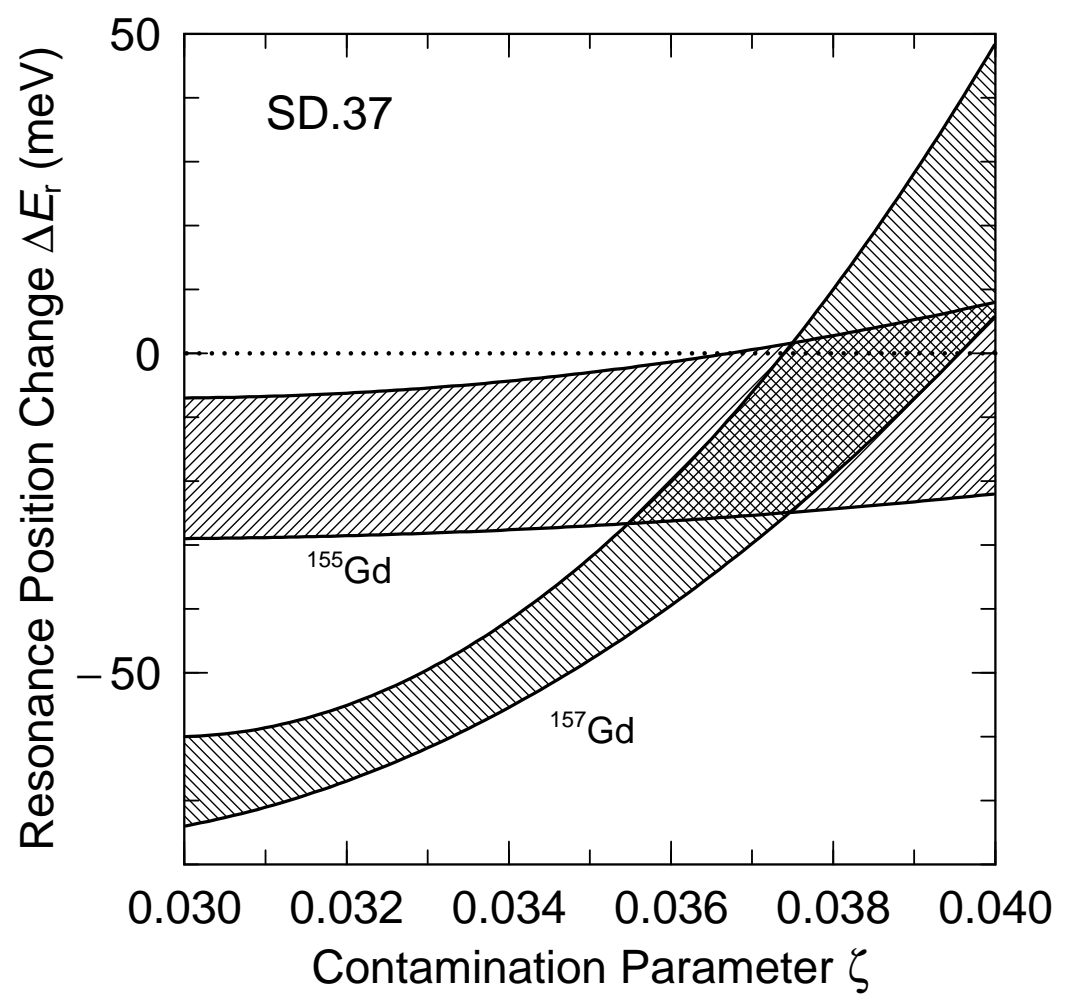

Figure 5: For the sample SD.37 we plot the resonance-position change $\Delta E_{\mathrm{r}}$ for ${ }^{155} \mathrm{Gd}$ and ${ }^{157} \mathrm{Gd}$ versus the contamination parameter $\zeta$, the ratio of the post-reactor contamination to the natural abundance. The two bands show the range of $\Delta E_{\mathrm{r}}$ for the temperature range $200-400^{\circ} \mathrm{C}$, with the lower bound of each band corresponding to $T=200^{\circ} \mathrm{C}$ and the upper bound to $T=400^{\circ} \mathrm{C}$. From the location of the overlap region of the two bands we deduce that the contamination parameter $\zeta$ is in the range 0.0355 to 0.0403 and that the resonance-position change $\Delta E_{\mathrm{r}}$ is in the range $-26 \mathrm{meV}$ to $+9 \mathrm{meV}$.

through the area covering $\Delta E_{\mathrm{r}}=0$. For the right-branch ranges we obtain large positive values for $\Delta E_{\mathrm{r}}$. This branch is not consistent with the Sm data giving either a nearzero branch or a negative branch range for $\Delta E_{\mathrm{r}}$. The requirement that the $\mathrm{Sm}$ and $\mathrm{Gd}$ data give the same result for the time variability of $\alpha$ therefore only allows the near-zero branches for Sm and $\mathrm{Gd}$ as acceptable choices for $\Delta E_{\mathrm{r}}$.

For the assumed temperature range $200-400{ }^{\circ} \mathrm{C}$, the bands for ${ }^{155} \mathrm{Gd}$ and ${ }^{157} \mathrm{Gd}$ corresponding to the left-branch ranges do intersect each other for $\zeta$ in the range $0.036-0.040$, yielding the range $-26 \mathrm{meV}$ to $+9 \mathrm{meV}$ for $\Delta E_{\mathrm{r}}$, as shown in Fig. 5. We also find that the band for ${ }^{155} \mathrm{Gd}$ is rather flat whereas that for ${ }^{157} \mathrm{Gd}$ shows a rapid increase as $\zeta$ changes. For this reason the common intersection gives $\Delta E_{\mathrm{r}}$ staying rather close to zero, even if the assumed equality for the two $\Delta E_{\mathrm{r}}$ 's is somewhat modified. Consider the equality is changed by a factor two, for example, in either direction. We find that the common range for $\zeta$ stays near $3 \sim 4 \%$, while that for $\Delta E_{\mathrm{r}}$ is expanded nearly twice as large, still leaving the conclusion of the consistency with the Sm data basically unchanged.

Similar results are obtained for the other samples except for SF84-1492 for which no reasonable solution is obtained.

In Fig. 6 we summarize the ranges of $\zeta$ and $\Delta E_{\mathrm{r}}$ obtained for the four samples. It is 


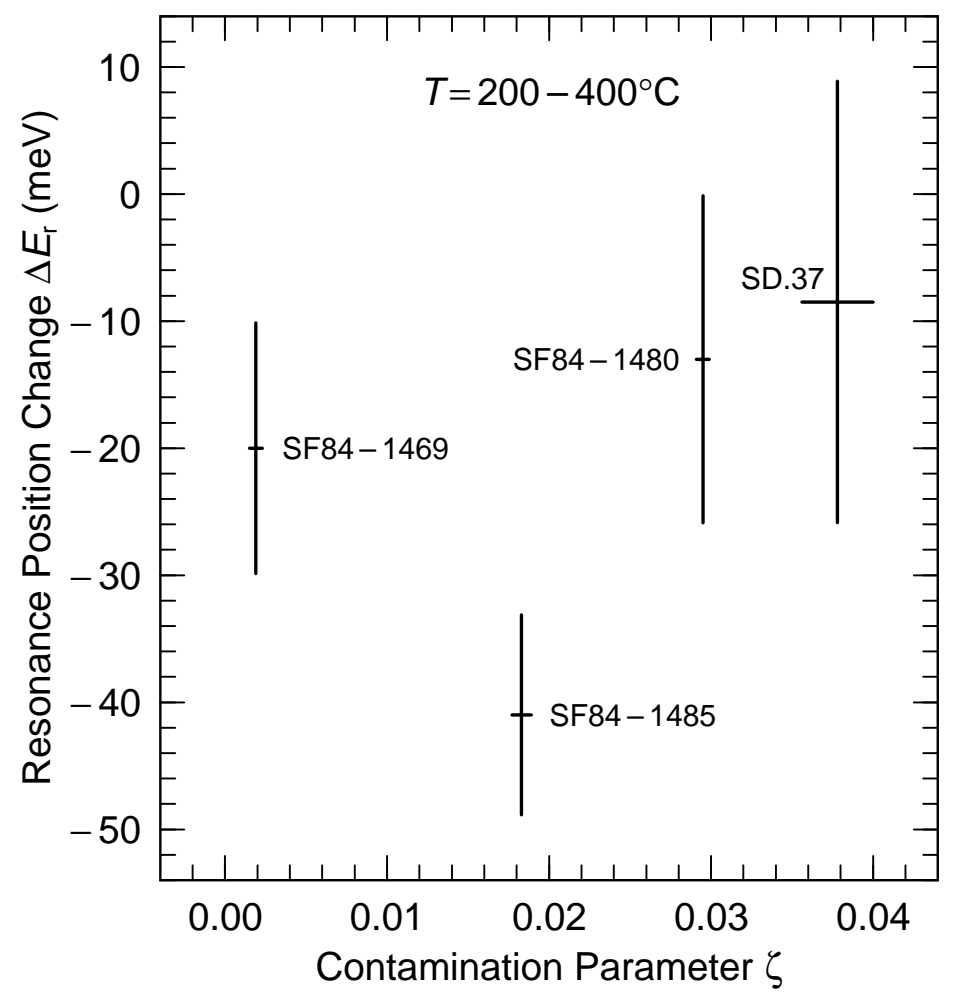

Figure 6: Summary of the ranges of $\Delta E_{\mathrm{r}}$ and $\zeta$ estimated from Gd data from four samples, for the assumed temperature range $T=200-400^{\circ} \mathrm{C}$.

remarkable to find that, with the exception of SF84-1485, the obtained values of $\Delta E_{\mathrm{r}}$ are consistent with the Sm result (27). The presence of contamination is clearly indicated and its magnitude is also consistent with the Sm data.

As for SF84-1485 and SF84-1492, one may suspect non-uniform contamination inside the reactor core or some other type of error. This suggests that more stringent limits on the variation of $\Delta E_{\mathrm{r}}$ would be achieved by collecting additional samples. It might be argued that assuming a common value of the contamination parameter $\zeta$ for the two isotopes is not well founded. However, it is the most simple assumption, and with this assumption the Sm and Gd results are entirely consistent with each other, both for the contamination parameter $\zeta$ and for the resonance-position change $\Delta E_{\mathrm{r}}$ for which the Sm and Gd mutually support the conclusion $\Delta E_{\mathrm{r}}=0$, though the conclusion is still tentative before the above plausibility assumptions are justified by more careful considerations, taking the need for more samples aside.

We also notice that, unlike in the Sm data, the result on Gd depends on the temperature rather sensitively. If we assume twice as wide the temperature range $200-600^{\circ} \mathrm{C}$, for example, the latitude of $\Delta E_{\mathrm{r}}$ in Fig. 6 extends roughly in the same rate. This tends to weaken the consistency check between the null results of Sm and Gd, but not to the extent that the non-null results are allowed to be mutually consistent. From this point of view, further improving the temperature estimate is strongly encouraged.

As an additional remark about the temperature, the same argument as in Sm showing that temperatures higher than $1000^{\circ} \mathrm{C}$ tend to constrain the cross sections to too small 
values compared with the measured values applies to $\mathrm{Gd}$ as well, though the result ought to be more complicated.

\section{Relation of the resonance-position change to the variation of the coupling constants}

Following Damour and Dyson we consider $\Delta E_{\mathrm{r}}$ as the change of the mass scale $\mathcal{M}_{\mathrm{c}}$ representing the difference of the expectation values of the Coulomb energy $H_{\mathrm{c}}$;

$$
\mathcal{M}_{\mathrm{c}}=<H_{\mathrm{c}}>_{1}-<H_{\mathrm{c}}>_{2}
$$

where the subscripts 1 and 2 refer to the excited state of ${ }^{150} \mathrm{Sm}$ and the ground state of ${ }^{149} \mathrm{Sm}$ plus a neutron, respectively. They estimated $\mathcal{M}_{c} \approx-1.1 \mathrm{MeV}$, which is proportional to $\alpha$ so that $[13]$

$$
\frac{\Delta \mathcal{M}_{\mathrm{c}}}{\mathcal{M}_{\mathrm{c}}}=\frac{\Delta \alpha}{\alpha}
$$

Identifying $\Delta \mathcal{M}_{\mathrm{c}}$ with (27) we obtain

$$
\frac{\dot{\alpha}}{\alpha}=(-0.2 \pm 0.8) \times 10^{-17} \mathrm{y}^{-1},
$$

which is about five times more stringent than in Ref. [13]. The improvement is mainly due to our choice of the separated right-branch range rather than the combined range.

If, on the other hand, we choose (28) corresponding to the left-branch range, we would obtain

$$
\frac{\dot{\alpha}}{\alpha}=(4.9 \pm 0.4) \times 10^{-17} \mathrm{y}^{-1}
$$

indicating an apparent evidence of the time-variability of $\alpha$.

We note that $E_{\mathrm{r}}=97.3 \mathrm{meV}$ of the resonance in question is extremely small compared with $\left|\mathcal{M}_{\mathrm{c}}\right| \approx 1 \mathrm{MeV}$, most part of which is cancelled by the contribution from the strong interaction $\mathcal{M}_{\mathrm{s}}$ defined analogously with $\mathcal{M}_{\mathrm{c}}$;

$$
\mathcal{M}_{\mathrm{c}} \approx-\mathcal{M}_{\mathrm{s}}
$$

We assume $\mathcal{M}_{\mathrm{s}}$ is proportional to $\alpha_{\mathrm{s}}^{n}$ with $n$ likely to be 1 , but allowing $n \neq 1$ anticipating some complication of nuclear physics. Combining this with (34) yields

$$
\frac{\Delta \alpha_{\mathrm{s}}}{\alpha_{\mathrm{s}}} \approx-\frac{1}{n} \frac{\Delta \alpha}{\alpha}
$$

if the change of the resonance energy is solely due to the change of the strong interaction coupling constant. In this way we expect simply the minus of (35) and (36) for the assumed value $n=1$. These are somewhat weaker than what we might find by assuming $\mathcal{M}_{\mathrm{s}} \approx 40 \mathrm{MeV}$, as suggested originally by Shlyakhter, who naively ignored the fact that the ground state ${ }^{149} \mathrm{Sm}$ has its own binding energy, which should be crucial for the near cancellation between the Coulomb and nuclear energies. 
In principle, one might argue that the nuclear energy levels should be expressed in terms of the more fundamental constant $\alpha_{\mathrm{QCD}}$. When applied to realistic low-energy phenomenology, however, it does not appear that this approach has reached the same level of rigor as QED. We instead expect to obtain a useful insight by expressing the result on the energy shift in terms of $\alpha_{\mathrm{s}}$, which has played important roles widely in understanding low-energy nuclear physics.

On the other hand, the argument can also be extended to a perhaps more realistic situation in which $\Delta E_{\mathrm{r}}$ is a combined effect both of the Coulomb and strong interactions. To show this we rewrite (37) without omitting $E_{\mathrm{r}}$;

$$
\mathcal{M}_{\mathrm{c}}+\mathcal{M}_{\mathrm{s}}=E_{\mathrm{r}}
$$

We also assume $n=1$, for the moment. By varying $\alpha$ and $\alpha_{\mathrm{s}}$ in (39), we obtain

$$
\frac{\Delta \alpha}{\alpha} \mathcal{M}_{\mathrm{c}}+\frac{\Delta \alpha_{\mathrm{s}}}{\alpha_{\mathrm{s}}} \mathcal{M}_{\mathrm{s}}=\Delta E_{\mathrm{r}}
$$

This is not enough to determine $\Delta \alpha / \alpha$ or $\Delta \alpha_{\mathrm{s}} / \alpha_{\mathrm{s}}$ separately. We need another relation between the two quantities. Here we simply introduce the ratio $u=\left(\Delta \alpha_{\mathrm{s}} / \alpha_{\mathrm{s}}\right) /(\Delta \alpha / \alpha)$ as a convenient parameter, in terms of which we solve (40) for $\Delta \alpha / \alpha$;

$$
\frac{\Delta \alpha}{\alpha}=\frac{\Delta E_{\mathrm{r}}}{(1-u) \mathcal{M}_{\mathrm{c}}+u E_{\mathrm{r}}}
$$

Unless $u$ is very close to 1 , we find

$$
(1-u) \frac{\Delta \alpha}{\alpha}=\left(\frac{1-u}{u}\right) \frac{\Delta \alpha_{\mathrm{s}}}{\alpha_{\mathrm{s}}}=\frac{\Delta E_{\mathrm{r}}}{\mathcal{M}_{\mathrm{c}}}
$$

hence giving $\dot{\alpha} / \alpha$ essentially of the same size as (35) or (36).

If, however,

$$
u-1 \ll \frac{E}{\mathcal{M}_{\mathrm{c}}} \sim \mathcal{O}\left(10^{-7}\right) \text {, }
$$

the denominator in (41) is almost $E_{\mathrm{r}}$, giving

$$
\frac{\Delta \alpha}{\alpha}=\frac{\Delta \alpha_{\mathrm{s}}}{\alpha_{\mathrm{s}}}=\frac{\Delta E_{\mathrm{r}}}{E_{\mathrm{r}}}
$$

which could be as large as 0.2 , resulting in $\dot{\alpha} / \alpha$ as large as $10^{-10} \mathrm{y}^{-1}$. The condition (43) implies, however, an unrealistic fine-tuning of parameters of any theoretical models.

\section{Discussions}

We have re-examined in detail the derivation of the upper bound on the shift with time of the resonance energy of ${ }^{149} \mathrm{Sm}$, and applied it to the new, carefully collected samples from deep-lying Oklo reactor zones. We reached the conclusion $\left|\Delta E_{\mathrm{r}}\right| \lesssim 16 \mathrm{meV}$. The surprising agreement to Shlyakhter's original result is probably somewhat accidental, since he in early 
years probably had access to few uncontaminated samples and since he also assumed the unrealistically low temperature $T=20^{\circ} \mathrm{C}$.

We still have another choice of nonzero shift $\Delta E_{\mathrm{r}} \sim-100 \mathrm{meV}$. From the former more favored choice, we derived $|\dot{\alpha} / \alpha| \approx\left|\dot{\alpha}_{\mathrm{s}} / \alpha_{\mathrm{s}}\right| \lesssim 10^{-17} \mathrm{y}^{-1}$, unless there is any reason why the equality $\dot{\alpha} / \alpha=\dot{\alpha}_{\mathrm{s}} / \alpha_{\mathrm{s}}$ holds true to an extreme accuracy of $10^{-7}$. The near equality for the time-variability of the two coupling constants is a consequence of the near cancellation between the two interactions resulting in an extremely small value of the resonance energies.

The cross section deduced from the 15 samples analyzed in Ref. [13] is $\hat{\sigma}_{149}=75 \pm 18 \mathrm{~kb}$ (two standard deviation). We find that the corresponding critical temperature for joining two ranges is $T_{\text {crit }} \approx 900^{\circ} \mathrm{C}$, which is sufficiently higher than their choice $T_{\text {upper }}=700^{\circ} \mathrm{C}$, implying that the two regions can be considered well separated. In this sense we may interpret their result in terms of the two ranges;

$$
-94 \pm 26 \mathrm{meV}, \quad \text { and } \quad 46 \pm 44 \mathrm{meV} .
$$

The separation and the sum of the half-widths are $140 \mathrm{meV}$ and $70 \mathrm{meV}$, respectively. The authors seemed nevertheless to favor an even more conservative attitude to consider as if the two ranges were connected, the far ends of which give

$$
-120 \mathrm{meV}<\Delta E<90 \mathrm{meV} \text {. }
$$

The limits might be narrowed slightly to $-115 \mathrm{meV}$ and $68 \mathrm{meV}$, respectively, at the level of one standard deviation, to be compared with our analysis. Notice also that $\Delta E_{\mathrm{r}}=0$ lies just on the edge of the two-standard deviation bound in the second of (45), indicating probably contamination of their samples.

We also studied the isotopic composition of $\mathrm{Gd}$ in Oklo, to look, for the first time, for a possible time-variation in the position of the low-lying resonances in ${ }^{155,157} \mathrm{Gd}$ and a corresponding time-variation of the coupling constants. We used the same methodology as in the studies of Sm. In the Gd analysis it was crucial to determine the amount of post-reactor contamination, in contrast to the case in the Sm analysis. By assuming that the energy-shifts of both isotopes of Gd are nearly the same having the same sign as that of Sm, the results of the Gd studies confirm and reinforce the conclusion of the null result based on the ${ }^{149} \mathrm{Sm}$ data. To consolidate this tentative but reasonable conclusion, further analyses might be required. This should include, in addition to collecting more samples, re-examining the proportionality between $\mathcal{M}_{\mathrm{c}}$ and $\alpha$ as assumed in [13], because any change in the Coulomb energy affects the size of a nucleus hence changing the kinetic energy as well, resulting in rather complicated dependence of the total energy on $\alpha$ in general. This aspect might be even more relevant when we add the contribution from the strong interaction.

We hope that we have shown with our detailed analysis here, having somewhat improved the result due to Damour and Dyson, that the constraints from the Oklo phenomenon should be considered to be quite realistic upper bounds on the time-variability of the fundamental constants. This result should provide valuable guidance in the search for viable unification models. 
The result obtained here, that the upper bounds on the rate-of-change of the fundamental constants are much lower than the value $\sim 10^{-10} \mathrm{y}^{-1}$ expected naturally in terms of the present age of the universe is likely linked to the cosmological constant problem, suggesting the presence of a small but nonzero $\Lambda$ [23-27]. One of the unique features of the approach in this paper, in contrast to the cosmological studies of the time-variation of the fundamental constants, is that we probe the time $\sim 2 \times 10^{9} \mathrm{y}$ ago. This is somewhat less distant than many of the cosmological phenomena considered so far, which have typical time scales of $\sim 10^{10} \mathrm{y}[3,5,8]$. The different time scales of the studies may be useful in trying to understand the recent finding that the fine-structure constant seems to exhibit a complicated time dependence [28].

We thank John Barrow for giving us important insight to the whole subject through his book [29] and for providing access to Ref. [7]. We are grateful to Thibault Damour for pointing out to us the importance of the effective cross section in the analysis of the Oklo phenomena.

The present work was partially supported by the REIMEI Research Resources of Japan Atomic Energy Research Institute and by the US Department of Energy, also in part by Grant-in-Aid for Scientific Research of the Ministry of Education, Science and Culture. 


\section{Appendices}

\section{A Numerical details}

The integral

$$
I_{c}=\int_{0}^{\infty} d E \sqrt{E} \exp (-E / T) \frac{E_{\gamma}}{\left(E-E_{\mathrm{r}}\right)^{2}+\Gamma_{\mathrm{tot}}^{2} / 4}
$$

occurring in Eq. (8) has to be evaluated by numerical quadrature. So that we can use Gauss-Laguerre integration we make the substitution $x=E / T$ and obtain

$$
I_{c}=T \int_{0}^{\infty} d x \sqrt{x T} \exp (-x) \frac{E_{\gamma}}{\left(x T-E_{\mathrm{r}}\right)^{2}+\Gamma_{\mathrm{tot}}^{2} / 4}=T \int_{0}^{\infty} d x \exp (-x) f_{1}(x) .
$$

where

$$
f_{1}(x)=\sqrt{x T} \frac{E_{\gamma}}{\left(x T-E_{\mathrm{r}}\right)^{2}+\Gamma_{\mathrm{tot}}^{2} / 4} .
$$

Because $f_{1}$ has a maximum at

$$
x_{\max }=\frac{E_{\mathrm{r}}+\sqrt{4 E_{\mathrm{r}}^{2}+3\left(\Gamma_{\mathrm{tot}} / 2\right)^{2}}}{4 T},
$$

it is necessary to divide the integration interval into two parts to achieve satisfactory

numerical accuracy. The first part of the integration should include the maximum of the function $f_{\mathrm{I}}$ and thus go from 0 to $x_{\mathrm{d}}$ where

$$
x_{\mathrm{d}}=2 x_{\max }
$$

is a suitable choice. However, the numerical results are very stable to reasonable variations of the choice of $x_{\mathrm{d}}$. Thus we rewrite

$$
\begin{aligned}
I_{\mathrm{c}} & =T \int_{0}^{x_{\mathrm{d}}} d x \sqrt{x T} \exp (-x) \frac{E_{\gamma}}{\left(x T-E_{\mathrm{r}}\right)^{2}+\Gamma_{\mathrm{tot}}^{2} / 4} \\
& +T \int_{x_{\mathrm{d}}}^{\infty} d x \sqrt{x T} \exp (-x) \frac{E_{\gamma}}{\left(x T-E_{\mathrm{r}}\right)^{2}+\Gamma_{\mathrm{tot}}^{2} / 4}
\end{aligned}
$$

So that we can use Gauss-Laguerre integration on the latter integral we substitute $y=$ $x-x_{\mathrm{d}}$ and obtain

$$
\begin{aligned}
I_{\mathrm{c}}= & T \int_{0}^{x_{\mathrm{d}}} d x \sqrt{x T} \exp (-x) \frac{E_{\gamma}}{\left(x T-E_{\mathrm{r}}\right)^{2}+\Gamma_{\text {tot }}^{2} / 4} \\
& +T \int_{0}^{\infty} d y \sqrt{\left(y+x_{\mathrm{d}}\right) T} \exp \left(-x_{\mathrm{d}}\right) \exp (-y) \frac{E_{\gamma}}{\left(\left(y+x_{\mathrm{d}}\right) T-E_{\mathrm{r}}\right)^{2}+\Gamma_{\mathrm{tot}}^{2} / 4}
\end{aligned}
$$

The first integral can now be calculated using Gauss-Legendre integration and the last integral can be calculated by Gauss-Laguerre integration. Thus we obtain for the numerical evaluation of Eq. A.1

$$
I_{\mathrm{c}}=\sum_{i=1}^{N_{\mathrm{G}}} \omega_{i}^{\mathrm{G}} F^{\mathrm{G}}\left(x_{i}^{\mathrm{G}}\right)+\exp \left(-x_{\mathrm{d}}\right) \sum_{i=1}^{N_{\mathrm{L}}} \omega_{i}^{\mathrm{L}} F^{\mathrm{L}}\left(y_{i}^{\mathrm{L}}\right)
$$


where the superscripts G and L indicate Gauss-Legendre and Gauss-Laguerre integration, respectively, and where

$$
F^{\mathrm{G}}(x)=T \sqrt{x T} \exp (-x) \frac{E_{\gamma}}{\left(x T-E_{\mathrm{r}}\right)^{2}+\Gamma_{\text {tot }}^{2} / 4}
$$

and

$$
F^{\mathrm{L}}(y)=T \sqrt{\left(y+x_{\mathrm{d}}\right) T} \frac{E_{\gamma}}{\left(\left(y+x_{\mathrm{d}}\right) T-E_{\mathrm{r}}\right)^{2}+\Gamma_{\mathrm{tot}}^{2} / 4}
$$

Note that the exponential term is retained in $F^{\mathrm{G}}$ but not in $F^{\mathrm{L}}$. 


\section{B Details of the fluence determination in the Oklo reactor zones}

In this section the following notation is used:

$N_{143} \quad$ The number of ${ }^{143} \mathrm{Nd}$ atoms per unit volume after the Oklo reactor shutdown. In the absence of contamination, in- and/or outflow of material this number is unchanged since the shutdown.

$N_{147}$ The number of ${ }^{147} \mathrm{Sm}$ atoms per unit volume after the Oklo reactor shutdown. In the absence of contamination, in- and/or outflow of material this number is unchanged since the shutdown.

$N_{235}$ The current number of ${ }^{235} \mathrm{U}$ atoms per unit volume in an Oklo reactor zone.

$N_{238}$ The current number of ${ }^{238} \mathrm{U}$ atoms per unit volume in an Oklo reactor zone.

$N_{235}^{0} \quad$ The number of ${ }^{235} \mathrm{U}$ atoms per unit volume in an Oklo reactor zone at the start of the reactor about two billion years ago. This number can simply be calculated from data observed now at other uranium deposits where no nuclear reaction has occurred.

$N_{238}^{0} \quad$ The number of ${ }^{238} \mathrm{U}$ atoms per unit volume in an Oklo reactor zone at the start of the reactor about two billion years ago. This number can simply be calculated from data observed now. at other uranium deposits where no nuclear reaction has occurred.

$I_{A} \quad$ Resonance integral.

$r_{\mathrm{rz}}$ Epi-thermal index. Accounts for the contribution of the neutron spectrum integrated over low-lying resonances. The subscript rz indicates that depends on the shape of the neutron spectrum and other factors and is therefore different in each reactor zone (rz) at Oklo.

$Y_{143} \quad$ Cumulative fission yield of ${ }^{143} \mathrm{Nd}$.

$Y_{147} \quad$ Cumulative fission yield of ${ }^{147} \mathrm{Sm}$.

$\hat{\sigma}_{\text {f235 }} \quad{ }^{235} \mathrm{U}$ fission cross section.

$\hat{\sigma}_{143}{ }^{143} \mathrm{Nd}$ total neutron-absorption cross section.

$\hat{\sigma}_{147} \quad{ }^{147} \mathrm{Sm}$ total neutron-absorption cross section.

$\hat{\sigma}_{235} \quad{ }^{235} \mathrm{U}$ total neutron-absorption cross section.

$\hat{\sigma}_{238}{ }^{238} \mathrm{U}$ total neutron-absorption cross section.

$\sigma_{A}^{0} \quad$ Thermal neutron-capture cross section. 
$\lambda_{235} \quad{ }^{235} \mathrm{U}$ decay constant.

$\lambda_{238} \quad{ }^{238} \mathrm{U}$ decay constant.

$\hat{\phi}_{\mathrm{rz}} \quad$ Average neutron flux in an Oklo reactor zone.

$C_{\mathrm{rz}} \quad$ Restitution factor of ${ }^{235} \mathrm{U}$ in an Oklo reactor zone. It accounts for the restitution of ${ }^{235} \mathrm{U}$ through neutron capture on ${ }^{238} \mathrm{U}, \beta$-decay of ${ }^{239} \mathrm{U}$ to ${ }^{239} \mathrm{~Np}$, $\beta$-decay of ${ }^{239} \mathrm{~Np}$ to ${ }^{239} \mathrm{Pu}, \alpha$-decay of ${ }^{239} \mathrm{Pu}$ to ${ }^{235} \mathrm{U}$, and smaller contributions from other reactions.

$\Delta t_{\mathrm{rz}} \quad$ Time duration of the neutron flux in the Oklo reactor.

$t$ Time elapsed since the start of the Oklo reactor, about 2 billion years.

The fluence $\hat{\phi}_{\mathrm{rz}} \Delta t_{\mathrm{rz}}$ in the different Oklo reactor zones may be determined from studies of the remnants of the reactions:

$$
\begin{array}{r}
{ }^{143} \mathrm{Nd}(\mathrm{n}, \gamma){ }^{144} \mathrm{Nd} \\
{ }^{147} \mathrm{Sm}(\mathrm{n}, \gamma){ }^{148} \mathrm{Sm} \\
{ }^{235} \mathrm{U}(\mathrm{n}, x) X \text { and } \\
{ }^{238} \mathrm{U}(\mathrm{n}, x) X
\end{array}
$$

The absorption cross sections $\hat{\sigma}_{143}$ and $\hat{\sigma}_{147}$ associated with the first two reactions may be expressed in the form

$$
\hat{\sigma}_{A}=\sigma_{A}^{0}+r_{\mathrm{rz}} I_{A}
$$

Specifically [29]

$$
\begin{aligned}
& \hat{\sigma}_{143}=335-100 r_{\mathrm{rz}} \\
& \hat{\sigma}_{147}=52+600 r_{\mathrm{rz}}
\end{aligned}
$$

in units of barn. The differential equations for the time-dependence of the number of atoms per unit volume $N_{A}$ of the isotopes involved in these reactions are

$$
\begin{aligned}
& \frac{d N_{143}(t)}{d t}=-\hat{\sigma}_{143} \hat{\phi}_{\mathrm{rz}} N_{143}(t)+N_{235}(t) Y_{143} \hat{\sigma}_{\mathrm{f} 235} \hat{\phi}_{\mathrm{rz}} \\
& \frac{d N_{147}(t)}{d t}=-\hat{\sigma}_{147} \hat{\phi}_{\mathrm{rz}} N_{147}(t)+N_{235}(t) Y_{147} \hat{\sigma}_{\mathrm{f} 235} \hat{\phi}_{\mathrm{rz}} \\
& \frac{d N_{235}(t)}{d t}=-\lambda_{235} N_{235}(t)+N_{235}(t)\left(1-C_{\mathrm{rz}}\right) \hat{\sigma}_{235} \hat{\phi}_{\mathrm{rz}} \\
& \frac{d N_{238}(t)}{d t}=-\lambda_{238} N_{238}(t)+N_{238}(t) \hat{\sigma}_{238} \hat{\phi}_{\mathrm{rz}}
\end{aligned}
$$


We obtain as solutions

$$
\begin{gathered}
N_{143}=\frac{N_{235}^{0} Y_{143} \hat{\sigma}_{\mathrm{f} 235}}{\hat{\sigma}_{143}-\left(1-C_{\mathrm{rz}}\right) \hat{\sigma}_{235}}\left\{\exp \left[-\left(1-C_{\mathrm{rz}}\right) \hat{\sigma}_{235} \hat{\phi}_{\mathrm{rz}} \Delta t_{\mathrm{rz}}\right]-\exp \left(-\hat{\sigma}_{143} \hat{\phi}_{\mathrm{rz}} \Delta t_{\mathrm{rz}}\right)\right\} \\
N_{147}=\frac{N_{235}^{0} Y_{147} \hat{\sigma}_{\mathrm{f} 235}}{\hat{\sigma}_{147}-\left(1-C_{\mathrm{rz}}\right) \hat{\sigma}_{235}}\left\{\exp \left[-\left(1-C_{\mathrm{rz}}\right) \hat{\sigma}_{235} \hat{\phi}_{\mathrm{rz}} \Delta t_{\mathrm{rz}}\right]-\exp \left(-\hat{\sigma}_{147} \hat{\phi}_{\mathrm{rz}} \Delta t_{\mathrm{rz}}\right)\right\} \\
\left(\frac{N_{235}}{N_{238}}\right)=\left(\frac{N_{235}^{0}}{N_{238}^{0}}\right) \exp \left[-\left(\lambda_{235}-\lambda_{238}\right) t\right] \exp \left\{-\left[\left(1-C_{\mathrm{rz}}\right) \hat{\sigma}_{235}-\hat{\sigma}_{238}\right] \hat{\phi}_{\mathrm{rz}} \Delta t_{\mathrm{rz}}\right\}
\end{gathered}
$$

One may easily obtain the ratio $N_{235}^{0} / N_{238}^{0}$ of ${ }^{235} \mathrm{U}$ to ${ }^{238} \mathrm{U}$ at Oklo by observing that today the ratio in uranium mines is 0.007252 and use

$$
\left(\frac{N_{235}^{0}}{N_{238}^{0}}\right)\left[\frac{\exp \left(-\lambda_{235} t\right)}{\exp \left(-\lambda_{238} t\right)}\right]=0.007252
$$

This leads to

$$
\left(\frac{N_{235}}{N_{238}}\right)=0.007252 \exp \left\{-\left[\left(1-C_{\mathrm{rz}}\right) \hat{\sigma}_{235}-\hat{\sigma}_{238}\right] \hat{\phi}_{\mathrm{rz}} \Delta t_{\mathrm{rz}}\right\}
$$

for the ratio ${ }^{235} \mathrm{U}$ to ${ }^{238} \mathrm{U}$ observed at Oklo today.

For each reactor zone at Oklo there are three unknowns that have to be determined: the restitution factor $C_{\mathrm{rz}}$, the epi-thermal index $r_{\mathrm{rz}}$, and the neutron fluence $\hat{\phi}_{\mathrm{rz}} \Delta t_{\mathrm{rz}}$. They can now be obtained as solutions to the three coupled equations $(\bar{B} .12)$, (B.13), and (B.16). Conditions for the occurrence of fission processes are influenced by many factors, such as the U content, neutron-absorber elements, water content among others. This explains why we often find different neutron fluences in different reactors. 


\section{References}

1) Y. Fujii, Oklo Phenomenon Revisited - Strong Interaction Coupling Constant Time-Dependent? in Proceedings of International Workshop on Gravitation and Astrophysics, ICRR, University of Tokyo, 17-19 November, 1997.

2) P.A.M. Dirac, Proc. Roy. Soc. A165 (1938) 199.

3) F. Hoyle, Galaxies, Nuclei and Quasars, Heinemann (London), 1965.

4) F.J. Dyson, Phys. Rev. Lett. 19 (1967) 1291.

5) P.C.W. Davies, J. Phys. A5 (1972) 1296.

6) A.I. Shlyakhter, Nature 264 (1976) 340.

7) A.I. Shlyakhter, ATOMKI Report A/1 (1983), unpublished.

8) L.L. Cowie and A. Songalia, Ap. J. 453 (1995) 596.

9) A. Godone, et al, Phys. Rev. Lett. 71 (1993) 2364.

10) R.W. Hellings, et al, Phys. Rev. Lett. 51 (1983) 1609.

11) See, for example, The Oklo Phenomenon, Proc. of a Symposium, Libreville, June, 1975 (IAEA, Vienna, 1975).

12) Natural Fission Reactors, Proc. of a meeting on natural fission reactor, Paris, France, December, 1977 (IAEA, Vienna, 1978).

13) T. Damour and F. Dyson, Nucl. Phys. B480 (1996) 37.

14) Neutron Cross Sections, Volume I (Academic Press, New York, 1984).

15) M. Lucas, R. Hagemann, R. Naudet, C. Renson and C. Chevalier,Natural Fission Reactors, Proc. of a meeting on natural fission reactor, Paris, France, December, 1977 (IAEA, Vienna, 1978) p. 407.

16) JENDL Nuclear data library, URL http://wwwndc.tokai.jaer.go.jp/NuC/index.html.

17) H. Hidaka and P. Holliger, Geochim. Cosmochim. Acta 62 (1998), 89.

18) D. Curtis, T. Benjamin, A. Gancarz, R. Loss, K. Rosman, J. DeLaeter, J.E. Delmore and W.J. Maeck, Applied Geochemistry, 4 (1989) 49.

19) H. Hidaka and F. Gauthier-Lafaye, unpublished data (1998).

20) P. Holliger and C. Deviller, Earth Planet. Sci. Lett. 52(1981), 76.

21) F. Gauthier-Lafaye, F. Weber and H. Ohmoto, Econ. Geol. 84 (1989) 2286.

22) L. Pourcelot and F. Gauthier-Lafaye, Chem. Geol. 157] (1999), 155.

23) S. Perlmutter, G. Aldering, G. Goldhaber, R.A. Knop, P. Nugent, P.G. Castro, S. Deustua, S. Fabbro, A. Goobar, D.E. Groom, I. M. Hook, A.G. Kim, M.Y. Kim, J.C. Lee, N.J. Nunes, R. Pain, C.R. Pennypacker, R. Quimby, C. Lidman, R.S. Ellis, M. Irwin, R.G. McMahon, P. Ruiz-Lapuente, N. Walton, B. Schaefer, B.J. Boyle, A.V. Filippenko, T. Matheson, A.S. Fruchter, N. Panagia, H.J.M. Newberg, W.J. Couch, ApJ, to be published.

24) Y. Fujii and T. Nishioka, Phys. Rev. D42 (1990) 361.

25) Y. Fujii, M. Omote and T. Nishioka, Prog. Theor. Phys. 92 (1994), 521. 
26) Y. Fujii, Astrop. Phys. 5 (1996) 133.

27) Y. Fujii, $\Omega_{\Lambda}$ and a new type of dissipative structure, delivered at XXXIIIrd Rencontres de Moriond, Fundamental Parameters in Cosmology (Les Arcs, France, January 17-24, 1998) and papers cited therein.

28) J.K. Webb, V.V. Flambaum, C.W. Churchill, M.J. Drinkwater and J.D. Barrow, Phys. Rev. Lett. 82 (1999) 884.

29) J.D. Barrow and F.J. Tipler,The Anthropic Cosmological Principle, Clarendon Press, Oxford, 1986.

30) C.H. Westcott, Effective cross-section values for well-moderated thermal reactor spectra, CRRP-960 (1960) (A.E.C.L., Chalk River). 